INSTITUT NATIONAL DE RECHERCHE EN INFORMATIQUE ET EN AUTOMATIQUE

\title{
A Time Domain Characterization of 2-microlocal Spaces
}

\author{
Stéphane Seuret — Jacques Lévy Véhel
}

\section{$\mathbf{N}^{\circ} \mathbf{4 5 4 5}$}

Septembre 2002

THÈME 4 



\title{
A Time Domain Characterization of 2-microlocal Spaces
}

\author{
Stéphane Seuret, Jacques Lévy Véhel \\ Thème 4 - Simulation et optimisation \\ de systèmes complexes \\ Projets Fractales \\ Rapport de recherche $\mathrm{n}^{\circ} 4545$ - Septembre 2002 - 34 pages
}

\begin{abstract}
In a recent work, new functional spaces were introduced. These spaces characterize the fine local regularity of functions, and are defined through simple estimations on the pointwise values of the functions. In this work, we generalize their definition to the whole class of continuous functions, which allows us to reach every positive Hölder exponent.

We prove that these new spaces coincide with the classical 2-microlocal spaces. It is interesting to notice that 2-microlocal spaces are originally defined in the frequency domain.. We prove the equality between both spaces in most of the cases.

Using this result, we propose an algorithm able to estimate a part of the 2-microlocal frontier. From the 2-microlocal frontier can be extracted all the usual regularity exponents. Thus, as a by-product of the algorithm, robust estimators of, for instance, both the pointwise and the local exponents are obtained. Experiments on sampled data show that reasonnable accuracy is achieved even for "difficult" functions such as continuous but nowhere differentiable ones.
\end{abstract}

Key-words: global regularity, local regularity, 2-microlocal spaces, regularity estimation algorithm, Hölder exponents. 


\section{Une caractérisation temporelle des espaces 2-microlocaux}

Résumé : Dans des travaux récents, de nouveaux espaces fonctionnels caractérisant la régularité locale fine des fonctions ont été introduits. Ces espaces sont définis dans le domaine temporel par des estimations simples faites sur les valeurs ponctuelles des fonctions étudiées. Nous généralisons cette définition à l'ensemble des fonctions continues, et cela nous permettra d'atteindre tous les exposants d'Hölder positifs.

Nous prouvons que ces espaces coincident avec les classiques espaces 2-microlocaux dans la plupart des cas. Cette égalité est intéressantes car les espaces 2-microlocaux sont définis dans le domaine fréquentiel.

Nous utilisons la propriété d'égalité entre les espaces mentionnés pour construire un algorithme capable d'estimer la frontière 2-microlocale de signaux continus en des points

donnés. Cette frontière contient toutes les notions de régularité usuelles, et l'on récupère donc en particulier un algorithme capable d'estimer de manière robuste l'exposant ponctuel d'Hölder. Des exemples d'estimation sont donnés, où l'on constate la bonne précision des résultats obtenus, y compris dans le cas où le comportement de la fonction étudiée est très erratique.

Mots-clés : régularité globale, régularité locale, espace 2-microlocaux, algorithme d'estimation de régularité, exposants d'Hölder. 


\section{Contents}

1 Introduction $\quad 3$

2 The Functional Spaces $K_{x_{0}}^{s, s^{\prime}} \quad 4$

2.1 Classical regularity exponents . . . . . . . . . . . . . . . 4

2.2 2-microlocal Spaces: Definition and Properties . . . . . . . . . . 5

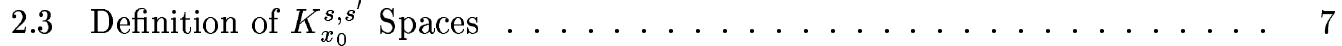

2.4 First Properties . . . . . . . . . . . . . . . . . . . 9

2.5 Domain of admissible exponents . . . . . . . . . . . . . . 13

3 Relation with 2-microlocal Spaces $\quad \mathbf{1 4}$

3.1 Main Result . . . . . . . . . . . . . . . . . . . . 15

3.2 Proof in a simple case . . . . . . . . . . . . . . . . 15

3.3 Applications . . . . . . . . . . . . . . . . . . . 18

4 Algorithms $\quad 23$

4.1 Background Ideas . . . . . . . . . . . . . . . . . . . . 23

4.2 Implementation . . . . . . . . . . . . . . . . . 23

5 Numerical Results $\quad \mathbf{2 4}$

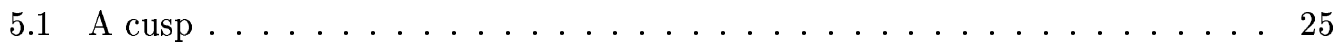

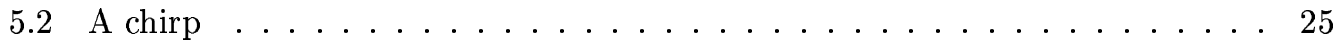

5.3 A sum of two chirps . . . . . . . . . . . . . 25

5.4 A Weierstrass function . . . . . . . . . . . . . . . 26

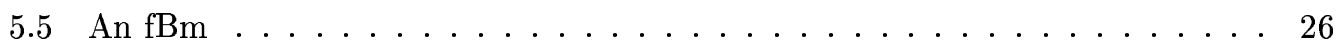

5.6 A generalized Weierstrass function $\ldots \ldots \ldots \ldots . \ldots . \ldots . \ldots 27$

6 Proof of Theorem 3.1 27

\section{Introduction}

The investigation of the regularity properties of functions has proved to be useful in many science domains, such as mathematics [2], signal processing [5], or physics.

Various notions of regularity exist. The easiest and most natural way of measuring the regularity of a function or a signal is given by the pointwise Hölder exponent. It is known that, although it is powerful and useful in many applications, this exponent does not fully characterize the behavior of a function at a given point. For instance, as is shown by the example of the chirp function, it does not take into account the oscillations around a point. One thus needs at least another regularity exponent to complete the description of the behavior of a function around a point $x_{0}$. Several choices have been proposed for this exponent, like the local Hölder exponent [12], or the chirp exponents introduced by Y. Meyer and S. Jaffard in [8] or [11]. 
All these exponents are contained in a larger frame, the so-called 2-microlocal analysis, introduced in [2] by J.M. Bony. The 2-microlocal spaces $C_{x_{0}}^{s, s^{\prime}}$ were first defined through a Littlewood-Paley analysis, and later characterized by a wavelet analysis. The price to pay for this deeper approach is a greater complexity in the definitions and the estimations of the parameters $s$ and $s^{\prime}$.

In [9], new functional spaces were introduced. These spaces, denoted by $K_{x_{0}}^{s, s^{\prime}}$, were defined for $0<s+s^{\prime}<1, s<1$, through simple conditions on the pointwise values of a real function $f$. This is sometimes an advantage, from a numerical point of view, over a definition by a Littlewood-Paley analysis. Indeed, instead of losing information by integrating (and thus by smoothing) sampled data, one uses every single point value. The main goals of this paper are to extend the definition of $K_{x_{0}}^{s, s^{\prime}}$ spaces to the case $s+s^{\prime} \geq 0, s^{\prime} \leq 0$, and to prove the equality $C_{x_{0}}^{s, s^{\prime}}=K_{x_{0}}^{s, s^{\prime}}$ when $s+s^{\prime} \notin \mathbb{N}$ and $s^{\prime}<0$. Thus $K_{x_{0}}^{s, s^{\prime}}$ spaces provide a time domain characterization of 2-microlocal spaces $C_{x_{0}}^{s, s^{\prime}}$ when the above conditions on $s$ and $s^{\prime}$ are completed. These restrictions correspond to the case of real-valued functions. Since the definition of $K_{x_{0}}^{s, s^{\prime}}$ spaces use pointwise values, we cannot yet hope to reach distributions.

After recalling the definitions of the classical regularity exponents, we deal in Section 2 with $K_{x_{0}}^{s, s^{\prime}}$ spaces, and with their basic properties. Section 3 contains the proof of the equality between $C_{x_{0}}^{s, s^{\prime}}$ and $K_{x_{0}}^{s, s^{\prime}}$ when $0<s+s^{\prime}<1$ and $s<1$. The general proof, a lot more technical, is given in Section 6 .

We propose in Section 4 an algorithm which uses this new characterization to estimate the 2-microlocal frontier of a continuous function. More precisely, given a signal $f$ and a point $x_{0}$, it estimates the frontier of the domain of all exponents $\left(s, s^{\prime}\right)$ such that $f \in K_{x_{0}}^{s, s^{\prime}}$ (or equivalently such that $f \in C_{x_{0}}^{s, s^{\prime}}$ ). Using this estimation of the frontier, the usual exponents are recovered. The results of this algorithm on different functions are presented in Section 5 .

\section{The Functional Spaces $K_{x_{0}}^{s, s^{\prime}}$}

\subsection{Classical regularity exponents}

Before introducing the $K_{x_{0}}^{s, s^{\prime}}$ spaces, we recall some usual notions of regularity.

Definition 2.1 Let $x_{0} \in \mathbb{R}, s$ a positive real number with $s \notin \mathbb{N}$, and $f$ a function: $\mathbb{R} \rightarrow \mathbb{R}$. We say that $f \in C_{x_{0}}^{s}$ if there exist a constant $C$ and a polynomial $P$ of degree smaller than $[s]$ such that

$$
\left|f(x)-P\left(x-x_{0}\right)\right| \leq C\left|x-x_{0}\right|^{s} .
$$

The pointwise Hölder exponent of $f$ at $x_{0}$ is defined by $\alpha_{p}\left(x_{0}\right)=\sup \left\{s: f \in C_{x_{0}}^{s}\right\}$. 
Definition 2.2 Let $x_{0} \in \mathbb{R}, 0<s<1$, and $f$ a function: $\mathbb{R} \rightarrow \mathbb{R}$. We say that $f \in$ $C_{l}^{s}\left(B\left(x_{0}, \rho\right)\right)$ if there exists a constant $C$ such that, for all $x, y$ in $B\left(x_{0}, \rho\right)$,

$$
|f(x)-f(y)| \leq C|x-y|^{s} .
$$

If $m<s<m+1(m \in \mathbb{N})$, then $f \in C_{l}^{s}\left(B\left(x_{0}, \rho\right)\right)$ means that there exists a constant $C$ such that, for all $x, y$ in $B\left(x_{0}, \rho\right),\left|\partial^{m} f(x)-\partial^{m} f(y)\right| \leq C|x-y|^{s-m}$.

Set now $\alpha_{l}\left(x_{0}, B\left(x_{0}, \rho\right)\right)=\sup \left\{s / f \in C_{l}^{s}\left(B\left(x_{0}, \rho\right)\right)\right\}$. The local Hölder exponent of $f$ at $x_{0}$, denoted by $\alpha_{l}\left(x_{0}\right)$, is defined by

$$
\alpha_{l}\left(x_{0}\right)=\lim _{\rho \rightarrow 0} \alpha_{l}\left(x_{0}, B\left(x_{0}, \rho\right)\right) .
$$

This exponent is well defined, since $\alpha_{l}\left(x_{0}, B\left(x_{0}, \rho\right)\right)$ is clearly a non-increasing function of $\rho$. Note finally that one always has $\alpha_{l}\left(x_{0}\right) \leq \alpha_{p}\left(x_{0}\right)$.

These definitions lead to different exponents, as shown by the two famous following examples:

- the cusp function $f(x)=|x|^{\alpha}$ : in this case, both Hölder exponents at 0 are equal to $\alpha$.

- the chirp function $f(x)=|x|^{\alpha} \sin \left(\frac{1}{\mid x^{\beta}}\right)$ : here the pointwise exponent at 0 is $\alpha$, while the local exponent at 0 is $\frac{\alpha}{1+\beta}$.

This difference is due to the fact that the local Hölder exponent takes into account the whole local behavior of the function $f$ around $x_{0}$, while the pointwise does not. In particular, the infinite oscillations around $x_{0}$ in the case of the chirp are considered as a special behavior by $\alpha_{l}\left(x_{0}\right)$, while they are ignored by $\alpha_{p}\left(x_{0}\right)$. More details on the relations between $\alpha_{l}$ and $\alpha_{p}$ can be found in [12].

Unfortunately one does not fully describe the regularity of a given function $f$ by the knowledge of those two exponents. Obviously there exist functions which have the same pointwise and local exponents at $x_{0}$, although they have a different behavior at this point. For example take $f_{1}(x)=|x|^{0.5} \sin \left(\frac{1}{|x|^{1}}\right)$ and $f_{2}(x)=|x|^{0.8} \sin \left(\frac{1}{x^{2.2}}\right)+|x|^{0.5} \sin \left(\frac{1}{x^{0.25}}\right)$. One checks that for both functions, $\alpha_{p}=0.5$ and $\alpha_{l}=0.25$, but their behaviors at 0 are different. A deeper approach and a more complete description of regularity are thus needed to go further.

\subsection{2-microlocal Spaces: Definition and Properties}

2-microlocal spaces $C_{x_{0}}^{s, s^{\prime}}$ were introduced by J.M. Bony in [2] in the PDE's frame, through Littlewood-Paley analysis. Let us quickly recall the definition of $C_{x_{0}}^{s, s^{\prime}}$.

Let $\phi$ be a function that belongs to the Schwartz space $\mathcal{S}(\mathbb{R})=\left\{f \in C^{\infty}(\mathbb{R}) / \forall(\gamma, \delta) \in\right.$ $\left.\mathbb{N}^{2}, \sup _{x}\left|x^{\gamma} \partial^{\delta} f(x)\right|<\infty\right\}$, such that the Fourier transform of $\phi$ satisfies

$$
\begin{array}{lll}
\hat{\phi}(\xi)=1 & \text { if } & |\xi| \leq 1 / 2 \\
\hat{\phi}(\xi)=0 & \text { if } & |\xi| \geq 1 .
\end{array}
$$


One defines $\phi_{j}(x)=2^{j} \phi\left(2^{j} x\right)$, and $\psi_{j}=\phi_{j+1}-\phi_{j}$. Let $f$ be a tempered distribution, thus belonging to the space $\mathcal{S}^{\prime}(\mathbb{R})$ defined by

$$
\mathcal{S}^{\prime}(\mathbb{R})=\left\{f / \exists C, \exists q \in \mathbb{N}, \forall g \in \mathcal{S}(\mathbb{R}),|\langle f, g\rangle| \leq C \pi_{q}(g)\right\},
$$

(where $\pi_{q}(g)=\sup \left\{(1+|x|)^{q}\left|\partial^{\delta} g(x)\right| /|\delta| \leq q, x \in \mathbb{R}\right\}$ ). The Littlewood-Paley analysis of $f$ is the set of distributions $\left\{S_{0} f, \Delta_{j} f\right\}_{j \geq 0}$, where

$$
S_{0} f=\phi * f \text { and } \Delta_{j} f=\psi_{j} * f .
$$

One has the fundamental decomposition (see [11] for details)

$$
f=S_{0} f+\sum_{j=0}^{+\infty} \Delta_{j} f
$$

We are now able to define the 2-microlocal spaces.

Definition 2.3 Let $x_{0} \in \mathbb{R}$ and $\left(s, s^{\prime}\right)$ two real numbers. A distribution $f \in \mathcal{S}^{\prime}(\mathbb{R})$ is said to belong to $C_{x_{0}}^{s, s^{\prime}}$ if there exists a constant $C$ such that

$$
\begin{aligned}
\left|S_{0} f(x)\right| & \leq C\left(1+\left|x-x_{0}\right|\right)^{-s^{\prime}} \\
\left|\Delta_{j} f(x)\right| & \leq C 2^{-j s}\left(1+2^{j}\left|x-x_{0}\right|\right)^{-s^{\prime}}
\end{aligned}
$$

This will provide us with a generalization of the notion of regularity at a point $x_{0}$, as we shall see later.

A useful characterization of $C_{x_{0}}^{s, s^{\prime}}$ is given by the wavelet coefficients of $f$ [7]. Indeed, let us take a function $\psi$ in the Schwartz class $\mathcal{S}(\mathbb{R})$, such that $\left\{\psi_{j, k}(x)=2^{j / 2} \psi\left(2^{j} x-k\right)\right\}_{(j, k) \in \mathbb{R}^{2}}$ forms an orthonormal basis of $L^{2}(\mathbb{R})$ (see for example [10]). The wavelet coefficients of $f$ are defined by (note that we do not use an $L^{2}$ normalization factor for the wavelet coefficients, and take $2^{j}$ instead of $2^{j / 2}$, for convenience in the following proofs)

$$
d_{j, k}=\int f(x) 2^{j} \psi\left(2^{j} x-k\right) d x .
$$

Moreover, if $\Psi$ is an admissible analyzing wavelet, the continuous wavelet transform is defined by

$$
W_{f}(a, b)=\frac{1}{a} \int f(x) \Psi\left(\frac{x-b}{a}\right) d x .
$$

Theorem 2.1 [8] Let $s, s^{\prime}$ be two real numbers. Let us assume that

- $\psi$ and $\Psi$ have $N$ vanishing moments, with $N>\max \left(s, s+s^{\prime}\right)$ 
- $\psi$ and $\Psi$ are in $\mathcal{S}(\mathbb{R})$ (the precise contraints on the regularity of the wavelets are explicited in [11]).

Then, the three following conditions are equivalent ones

1. $f \in C_{x_{0}}^{s, s^{\prime}}$,

2. $\forall j, k$ such that $\left|x_{0}-k 2^{-j}\right| \leq 1,\left|d_{j, k}\right| \leq C 2^{-j s}\left(1+\left|k-2^{j} x_{0}\right|\right)^{-s^{\prime}}$,

3. $\forall a>0,\left|b-x_{0}\right|<1,\left|W_{f}(a, b)\right| \leq C a^{s}\left(1+\frac{\left|b-x_{0}\right|}{a}\right)^{-s^{\prime}}$.

There also exists a wavelet characterization of the pointwise Hölder exponent, due to S. Jaffard ([8])

Theorem 2.2 Assume that $f \in C^{s}\left(x_{0}\right)$. If $\left|k 2^{-j}-x_{0}\right| \leq 1 / 2$, then

$$
\left|d_{j, k}\right| \leq C 2^{-s j}\left(1+2^{j}\left|k 2^{-j}-x_{0}\right|\right)^{s} .
$$

Conversely, if (7) holds for all $(j, k)$ 's such that $\left|k 2^{-j}-x_{0}\right| \leq 2^{-j /(\log j)^{2}}$, and if $f \in C^{\log }$, then there exist a constant $C$ and a polynomial $P$ of degree at most $[s]$ such that

$$
\left|f(x)-P\left(x-x_{0}\right)\right| \leq C\left|x-x_{0}\right|^{s}\left(\log \left(\left|x-x_{0}\right|\right)\right)^{2} .
$$

$C^{l o g}$ is the class of functions $f$ whose wavelet coefficients verify $\left|d_{j, k}\right| \leq C 2^{-\frac{j}{\log j_{j}}}$. This regularity condition is stronger than uniform continuity, but does not imply a uniform Hölder continuity.

\subsection{Definition of $K_{x_{0}}^{s, s^{\prime}}$ Spaces}

$K_{x_{0}}^{s, s^{\prime}}$ spaces were defined in [9] for nowhere differentiable functions. We extend here this definition to a wider range of exponents

Definition 2.4 Let $x_{0} \in \mathbb{R}$, and $s, s^{\prime}$ be two real numbers satisfying $s^{\prime} \leq 0$ and $s+s^{\prime} \geq 0$ (and thus $s \geq 0$ ).

Let $m=\left[s+s^{\prime}\right]$. A function $f: \mathbb{R} \rightarrow \mathbb{R}$ is said to belong to $K_{x_{0}}^{s, s^{\prime}}$ if there exist $0<\delta<1 / 4$, a polynomial $P$ of degree smaller than $[s]-m$, and a constant $C$, that verify

$$
\left|\frac{\partial^{m} f(x)-P(x)}{\left|x-x_{0}\right|^{[s]-m}}-\frac{\partial^{m} f(y)-P(y)}{\left|y-x_{0}\right|^{[s]-m}}\right| \leq C|x-y|^{s+s^{\prime}-m}\left(|x-y|+\left|x-x_{0}\right|\right)^{-s^{\prime}-[s]+m}
$$

for all $x, y$ such that $0<\left|x-x_{0}\right|<\delta, 0<\left|y-x_{0}\right|<\delta$.

Let us make a few remarks on this definition.

- If $s+s^{\prime}<1$ and $s<1$ (i.e. $m=[s]=0$ ), then the original definition of [9] is recovered

$$
|f(x)-f(y)| \leq C|x-y|^{s+s^{\prime}}\left(|x-y|+\left|x-x_{0}\right|\right)^{-s^{\prime}} .
$$


- If $m<s+s^{\prime}<m+1$ and $s<m+1$ (i.e. $[s]=m$ ), one obtains a simpler formulation of the definition

$$
\left|\partial^{m} f(x)-\partial^{m} f(y)\right| \leq C|x-y|^{s-m+s^{\prime}}\left(|x-y|+\left|x-x_{0}\right|\right)^{-s^{\prime}} .
$$

- The right term in the above inequality seems to be asymmetric, but it is not. Remarking that $\left(|x-y|+\left|x-x_{0}\right|\right) \leq 2\left(|x-y|+\left|y-x_{0}\right|\right)$, this right term of (9) can be re-written as one of the two following expressions (the last one being symmetric in $x$ and $y$ )

$$
\begin{gathered}
|x-y|^{s-m+s^{\prime}}\left(|x-y|+\left|y-x_{0}\right|\right)^{-s^{\prime}-[s]+m} \\
|x-y|^{s-m+s^{\prime}}\left(\left(|x-y|+\left|x-x_{0}\right|\right)\left(|x-y|+\left|y-x_{0}\right|\right)\right)^{\left(-s^{\prime}-[s]+m\right) / 2}
\end{gathered}
$$

- In the following, we will most of the time avoid the critical cases $s+s^{\prime} \in \mathbb{I}$ and $s \in \mathbb{N}$. Indeed, they would require the use of Zygmund spaces instead of the usual homogeneous Hölder spaces $C^{\alpha}(\mathbb{R})$.

- If $g_{m}(x)$ denotes the function $\frac{\partial^{m} f(x)-P(x)}{\mid x-x_{0}[s]-m}$, one easily sees that $g_{m}$ is at least continuous at each point, especially at $x_{0}$. Indeed, if $-s^{\prime}-[s]+m>0$, one writes

$$
\left|g_{m}(x)-g_{m}(y)\right| \leq C|x-y|^{s+s^{\prime}-m}
$$

with $0<s+s^{\prime}-m<1$. Thus $g_{m} \in C^{s+s^{\prime}-m}$ around $x_{0}$. On the other hand, if $-s^{\prime}-[s]+m \leq 0$, one has

$$
\left|g_{m}(x)-g_{m}(y)\right| \leq C|x-y|^{s-[s]}\left(\frac{|x-y|}{|x-y|+\left|x-x_{0}\right|}\right)^{s^{\prime}+[s]-m} \leq|x-y|^{s-[s]}
$$

with $0<s-[s]<1$, thus $g_{m} \in C^{s-[s]}$ around $x_{0}$.

One is thus allowed to take $y=x_{0}$ in (9) or in (10), and also to consider the real number $g_{m}\left(x_{0}\right)$.

- The left hand-side of (9) and the exponents we use may seem complex. The necessity of the different terms is however easy to understand: one tries to reduce the study of $f$ to the one of a new function derived from $f$ that will belong to some $K_{x_{0}}^{t, t^{\prime}}$ with $0 \leq t+t^{\prime}<1$ and $0 \leq t<1$.

In a way, we partition the subset of $\mathbb{R}^{2}\left\{\left(s, s^{\prime}\right) / s+s^{\prime} \geq 0, s^{\prime} \leq 0\right.$ and $\left.s>0\right\}$ into tiles of same size, and we translate the problem to the "initial" tile $\left\{\left(s, s^{\prime}\right) / 0<s+s^{\prime}<\right.$ 1 and $s<1$ \} (see figure 1 ).

For example, if a function $f$ belongs to $K_{x_{0}}^{s, s^{\prime}}$ with $m \leq s+s^{\prime}<m+1, f$ admits around $x_{0}$ a derivative of order $m$. The formula simply says that $f \in K_{x_{0}}^{s, s^{\prime}}$ means that 


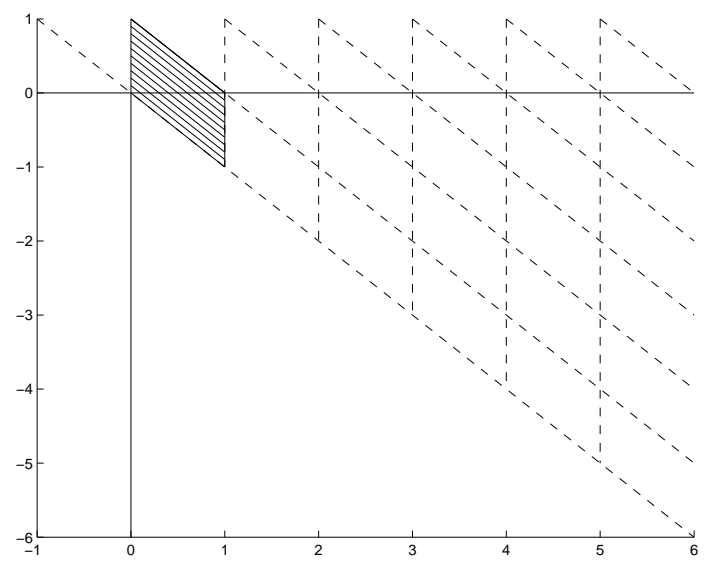

Figure 1: Paving of the half plane $s>0$.

$\partial^{m} f \in K_{x_{0}}^{s-m, s^{\prime}}$. Then, if $f \in K_{x_{0}}^{s, s^{\prime}}$ with $0<s+s^{\prime}<1$ with $s>1$, one replaces $f$ in formula (10) by $\frac{f(x)-P(x)}{\left.\left|x-x_{0}\right| s\right]}$, and this new function will belong to $K_{x_{0}}^{s-[s], s^{\prime}+[s]}$.

One can formalize these thoughts by saying that the operator

$$
f \rightarrow \frac{\partial^{m} f(x)-P(x)}{\left|x-x_{0}\right|^{[s]-m}}
$$

(for the correct polynomial $P$ ) maps $K_{x_{0}}^{s, s^{\prime}}$ into $K_{x_{0}}^{s-[s], s^{\prime}+[s]-m}$.

\subsection{First Properties}

We stress several interesting properties of these spaces $K_{x_{0}}^{s, s^{\prime}}$. Propositions (2.1) to (2.4) are just extensions of the corresponding ones in [9] to the general case.

The first proposition is an embedding property between the spaces $K_{x_{0}}^{s, s^{\prime}}$.

Proposition 2.1 Let $x_{0} \in \mathbb{R}$, and $s, s^{\prime}, t, t^{\prime}$ be four real numbers such that $t \leq s$ and $t+t^{\prime} \leq s+s^{\prime}$. Then $K_{x_{0}}^{s, s^{\prime}} \subset K_{x_{0}}^{t, t^{\prime}}$.

The proof of Proposition 2.1 is splitted into two simpler lemmas

Lemma 2.1 Let $x_{0} \in \mathbb{R}$, and $s, s^{\prime}, t, t^{\prime}$ be four real numbers such that $t \leq s$ and $t+t^{\prime}=s+s^{\prime}$. Then $K_{x_{0}}^{s, s^{\prime}} \subset K_{x_{0}}^{t, t^{\prime}}$.

Proof: Let us treat the case $s+s^{\prime}<1$ (i.e. $m=0$ ), the general case is then deduced by replacing $f$ by $\partial^{m} f$ and $s$ by $s-m$.

$\mathrm{RR} \mathrm{n}^{\circ} 4545$ 
If $[t]=[s]$, the result is obvious. Let us treat the case $[t]=[s]-1$, the general result will then easily follow by iteration.

Let us assume that $f \in K_{x_{0}}^{s, s^{\prime}}, x_{0}=0$ and that $|y| \leq|x|$, without loss of generality. One also assumes that $x>0$, by replacing $z \rightarrow f(z)$ by $z \rightarrow f(-z)$. There exists a polynomial $P$ such that (9) holds. One is now looking for a polynomial $P_{t}$ that satisfies

$$
\left|\frac{f(x)-P_{t}(x)}{|x|^{[s]-1}}-\frac{f(y)-P_{t}(y)}{|y|^{[s]-1}}\right| \leq C|x-y|^{t+t^{\prime}}(|x-y|+|x|)^{-t^{\prime}-[t]}
$$

Let us denote by $P_{t}$ the polynomial of degree $[t]=[s]-1$ with the same coefficients as $P$ up to degree $[t]$. To simplify the notations, let us define $g(x)=\frac{f(x)-P(x)}{|x|^{[s]}}$ and $g_{t}(x)=\frac{f(x)-P_{t}(x)}{|x|^{[s]-1}}$. Then,

$$
\forall x, \quad g_{t}(x)=|x| g(x)+\frac{P(x)-P_{t}(x)}{|x|^{[s]-1}},
$$

Now,

$$
\left|g_{t}(x)-g_{t}(y)\right| \leq\left|\left(|x| g(x)+\frac{P(x)-P_{t}(x)}{|x|^{[s]-1}}\right)-\left(|y| g(y)+\frac{P(y)-P_{t}(y)}{|y|^{\mid s]-1}}\right)\right|
$$

By construction, $P(x)-P_{t}(x)$ is a polynomial with only one non-zero coefficient. Thus $P(x)-P_{t}(x)=a x^{[s]}$, and

$$
\begin{aligned}
\left|g_{t}(x)-g_{t}(y)\right| & \leq|| x|g(x)-| y|g(y)|+a\left|\frac{x^{[s]}}{|x|^{[s]-1}}-\frac{y^{[s]}}{|y|^{[s]-1}}\right| \\
& \leq|| x|(g(x)-g(0))-| y|(g(y)-g(0))|+(|a|+|g(0)|)|x-y|
\end{aligned}
$$

A useful remark is that

$$
\begin{aligned}
|x-y| & =|x-y|^{t+t^{\prime}}|x-y|^{-t^{\prime}+1-t} \\
& \leq|x-y|^{t+t^{\prime}}(|x-y|+|x|)^{-t^{\prime}+1-t} \\
& \leq|x-y|^{t+t^{\prime}}(|x-y|+|x|)^{-t^{\prime}-[t]} .
\end{aligned}
$$

(note that $1-t>-[t]$ ). A direct upper bound for $g$ is obtained by taking $y=0$ in (9)

$$
\forall x,|g(x)-g(0)| \leq C|x|^{s-[s]} .
$$

Applying (13) and (9), the last term $(T)=|| x|(g(x)-g(0))-| y|(g(y)-g(0))|$ is treated as follows

$$
\begin{aligned}
(T) \leq & || x|-| y|| g(x)-g(0)|+||y|(g(x)-g(0))-|y|(g(y)-g(0)) \mid \\
\leq & |x-y||g(x)-g(0)|+|y||g(x)-g(y)| \\
\leq & C|x-y||x|^{s-[s]}+|y||x-y|^{s+s^{\prime}}(|x|+|x-y|)^{-s^{\prime}-[s]} \\
\leq & C|x-y|^{t+t^{\prime}}|x-y|^{1-\left(t+t^{\prime}\right)}|x|^{s-[s]} \\
& +|y||x-y|^{s+s^{\prime}}(|x|+|x-y|)^{-s^{\prime}-[s]}
\end{aligned}
$$

INRIA 
Since $s-[s]$ and $1-\left(t+t^{\prime}\right)$ are positive, one upper-bounds $|x|^{s-[s]},|x-y|^{1-\left(t+t^{\prime}\right)}$ and $|y|$ respectively by $(|x|+|x-y|)^{s-[s]},(|x|+|x-y|)^{1-\left(t+t^{\prime}\right)}$ and $(|x|+|x-y|)$. This gives, using $[t]=[s]-1$,

$$
\begin{aligned}
(T) \leq & C|x-y|^{t+t^{\prime}}(|x|+|x-y|)^{-t^{\prime}-[t]+s-t+2} \\
& +|x-y|^{s+s^{\prime}}(|x|+|x-y|)^{-s^{\prime}-[t]+2} .
\end{aligned}
$$

Eventually, remembering that $t+t^{\prime}=s+s^{\prime}$, one has $-s^{\prime}-[t]+2=-t^{\prime}-[t]+2+s-t$. One concludes, using $2+s-t>0$, that

$$
\left|g_{t}(x)-g_{t}(y)\right| \leq C|x-y|^{t+t^{\prime}}(|x|+|x-y|)^{-t^{\prime}-[t]}
$$

which gives exactly the required result.

Lemma 2.2 Let $x_{0} \in \mathbb{R}$, and $s, s^{\prime}, t^{\prime}$ be three real numbers such that $t^{\prime} \leq s^{\prime}$. Then $K_{x_{0}}^{s, s^{\prime}} \subset$ $K_{x_{0}}^{s, t^{\prime}}$.

Proof: If $\left[s+s^{\prime}\right]=\left[s+t^{\prime}\right]$, the result is obvious.

Assume that $m=\left[s+s^{\prime}\right]=\left[s+t^{\prime}\right]+1$, the general result will then easily follow. As usual now, we will assume without loss of generality that $x_{0}=0$ and $|y| \leq x$. By assumption, (9) holds, and one wants to prove

$$
\begin{array}{r}
\left|\frac{\partial^{m-1} f(x)-P(x)}{|x|^{[s]-(m-1)}}-\frac{\partial^{m-1} f(y)-P(y)}{|y|[s]-(m-1)}\right| \leq \\
C|x-y|^{s-(m-1)+t^{\prime}}(|x-y|+|x|)^{-t^{\prime}-[s]+(m-1)} .
\end{array}
$$

Taking $y=0$ in (9) yields

$$
\left|\partial^{m} f(x)-P(x)\right| \leq C|x|^{s-m}
$$

for a certain polynomial $P$ of degree at most $[s]-m$. Integrating first this last inequality between 0 and $x$, and then between $y$ and $x$, one obtains

$$
\left|\partial^{m-1} f(x)-P_{t^{\prime}}(x)\right| \leq C|x|^{s-m+1}
$$

and

$$
\left|\left(\partial^{m-1} f(x)-P_{t^{\prime}}(x)\right)-\left(\partial^{m-1} f(y)-P_{t^{\prime}}(y)\right)\right| \leq C|x-y||x|^{s-m}
$$

for a polynomial $P_{t^{\prime}}$ of degree at most $[s]-m+1$ (the same one for both (14) and (15)). One first writes

$$
\left|\frac{\partial^{m-1} f(x)-P_{t^{\prime}}(x)}{|x|^{[s]-m+1}}-\frac{\partial^{m-1} f(y)-P_{t^{\prime}}(y)}{|y|^{[s]-m+1}}\right| \leq(I)+(I I),
$$


where

$$
(I)=\left|\frac{\partial^{m-1} f(x)-P_{t^{\prime}}(x)}{|x|^{[s]-m+1}}-\frac{\partial^{m-1} f(y)-P_{t^{\prime}}(y)}{|x|^{[s]-m+1}}\right|
$$

and

$$
(I I)=\left|\frac{\partial^{m-1} f(y)-P_{t^{\prime}}(y)}{|x|^{[s]-m+1}}-\frac{\partial^{m-1} f(y)-P_{t^{\prime}}(y)}{|y|^{[s]-m+1}}\right| .
$$

Dividing (15) by $|x|^{[s]-m+1},(I)$ is bounded by $C|x-y||x|^{s-[s]-1}$. On the other hand, using (14), one has

$$
\begin{aligned}
(I I) & \leq\left|\partial^{m-1} f(y)-P_{t^{\prime}}(y)\right| \frac{1}{|x|^{[s]-m+1}}-\frac{1}{|y|^{[s]-m+1}} \mid \\
& \leq\left|\partial^{m-1} f(y)-P_{t^{\prime}}(y)\right| \frac{|x|^{[s]-m+1}-|y|^{[s]-m+1}}{|x y|^{[s]-m+1}} \\
& \leq\left|\partial^{m-1} f(y)-P_{t^{\prime}}(y)\right| \frac{|x-y||x|^{[s]-m}}{|x y|^{[s]-m+1}} \\
& \leq C|y|^{s-m+1} \frac{|x-y||x|^{-1}}{|y|^{[s]-m+1}} \leq C \frac{|y|^{s-[s]}}{|x|}|x-y| \\
& \leq C\left(\frac{|y|}{|x|}\right)^{s-[s]}|x|^{s-[s]-1}|x-y| \leq C|x-y||x|^{s-[s]-1},
\end{aligned}
$$

since $\frac{|y|}{x \mid}$ is bounded by 1 .

The same kind of manipulations of exponents (but easier) as at the end of the previous proposition can be performed. Remarking that $|x|^{-s^{\prime}} \leq C(|x-y|+|x|)^{-s^{\prime}}$ and using $0<s+t^{\prime}-(m-1)<1$, it is easily verified that

$$
\begin{aligned}
|x-y||x|^{s-[s]-1} & =|x-y|^{s+t^{\prime}-(m-1)}|x-y|^{1-\left(s+t^{\prime}-(m-1)\right)}|x|^{s-[s]-1} \\
& \leq|x-y|^{s+t^{\prime}-(m-1)}(|x-y|+|x|)^{1-\left(s+t^{\prime}-(m-1)\right)+s-[s]-1} \\
& \leq|x-y|^{s+t^{\prime}-(m-1)}(|x-y|+|x|)^{-t^{\prime}+(m-1)-[s]}
\end{aligned}
$$

which gives the result.

Combining Lemmas 2.1 and 2.1, Proposition 2.1 is proved.

We now compare these $K_{x_{0}}^{s, s^{\prime}}$ with the classical pointwise Hölder spaces.

Proposition 2.2 Let $x_{0} \in \mathbb{R}$, and $s$ be a real number such that $s>0, s \notin \mathbb{N}$. Then $C_{x_{0}}^{s}=K_{x_{0}}^{s,-s}$. 
Proof: Let $f$ be a function in $C_{x_{0}}^{s}$. One writes, using the approximating polynomial $P$ found in (1),

$$
\begin{aligned}
\left|\frac{f(x)-P(x)}{\left|x-x_{0}\right|^{[s]}}-\frac{f(y)-P(y)}{\left|y-x_{0}\right|^{[s]}}\right| & \leq\left|\frac{f(x)-P(x)}{\left|x-x_{0}\right|^{[s]}}\right|+\left|\frac{f(y)-P(y)}{\left|y-x_{0}\right|^{[s]}}\right| \\
& \leq C\left(\left|x-x_{0}\right|^{s-[s]}+\left|y-x_{0}\right|^{s-[s]}\right) \\
& \leq C\left(|x-y|+\left|x-x_{0}\right|\right)^{s-[s]} .
\end{aligned}
$$

This proves $f \in K_{x_{0}}^{s,-s}$.

On the other hand, let $f$ be a function in $K_{x_{0}}^{s,-s}$. Taking $y=x_{0}$ in (9) leads to (remember that $g_{m}(x)$ id defined by $\left.\frac{\partial^{m} f(x)-P(x)}{\left|x-x_{0}\right|^{[s]-m}}\right)$

$$
\left|g_{m}(x)-g_{m}(0)\right|=\left|\frac{\partial^{m} f(x)-P(x)}{\left|x-x_{0}\right|^{[s]-m}}-g_{m}(0)\right| \leq\left|x-x_{0}\right|^{s-[s]},
$$

thus $\left|\partial^{m} f(x)-P_{1}(x)\right| \leq C\left|x-x_{0}\right|^{s-m}$, where $P_{1}$ is a polynomial of order smaller than $[s]-m$. Last inequality can be reformulated in

$$
\left|\partial^{m}\left(f-P_{2}\right)(x)\right| \leq C\left|x-x_{0}\right|^{s-m},
$$

where $P_{2}$ is a polynomial of degree less than $[s]$. This reads $\partial^{m} f \in C_{x_{0}}^{s-m}$, which implies $f \in C_{x_{0}}^{s}$. Combining this with Proposition 2.2 leads to the result.

It is easily verified that $\forall s^{\prime} \leq 0$ with $s+s^{\prime}>0, K_{x_{0}}^{s, s^{\prime}} \subset C_{x_{0}}^{s}$, thus, for all $s^{\prime} \leq 0$, $K_{x_{0}}^{s, s^{\prime}} \subset C_{x_{0}}^{s} \subset K_{x_{0}}^{s,-s}$. As soon as a function belongs to a $K_{x_{0}}^{s, s^{\prime}}$, it automatically belongs to $C_{x_{0}}^{s}$. That also means, by reciprocity, that a function $f$ whose pointwise Hölder exponent is $s>0$ cannot belong to any $K_{x_{0}}^{t, t^{\prime}}$, for $t>s$, whatever $t^{\prime}$ is. This is an important property of $K_{x_{0}}^{s, s^{\prime}}$ spaces.

\subsection{Domain of admissible exponents}

Definition 2.5 Let $f$ be a function: $\mathbb{R} \rightarrow \mathbb{R}$. $E\left(f, x_{0}\right)$ denotes the set in the half plane $\left\{\left(s, s^{\prime}\right): s+s^{\prime} \geq 0, s^{\prime} \leq 0\right.$ and $\left.s>0\right\}$ of all couples $\left(s, s^{\prime}\right)$ such that $f \in K_{x_{0}}^{s, s^{\prime}}$.

Let us notice that this set is convex by Proposition 2.1. Moreover, it cannot intersect the open half-space defined by $\left\{\left(s, s^{\prime}\right): s>\alpha_{p}(f)\right\}$, because of the last remark in the previous section. Eventually, if $\Gamma$ denotes the boundary of $E\left(f, x_{0}\right)$, it is easily shown that $\Gamma$ is, again by propositions 2.1 and 2.2, the graph of a function $s=\gamma\left(s^{\prime}\right) . \gamma$ is concave, decreasing, with slope greater than -1 .

The following proposition links the $K_{x_{0}}^{s, s^{\prime}}$ spaces with the local Hölder exponent defined in the first section 
Proposition 2.3 Let $x_{0} \in \mathbb{R}$, and $f: \mathbb{R} \rightarrow \mathbb{R}$ such that $\gamma(0)>0$. The local Hölder exponent corresponds to the intersection of the frontier of the domain with the $x$-axis, i.e. $\alpha_{l}=\gamma(0)$.

Proof: From the definition of $K_{x_{0}}^{s, 0}$, one obtains, for $\left|x-x_{0}\right|<\delta,\left|y-x_{0}\right|<\delta$, (remark that $s^{\prime}=0$ implies $[s]=m$ )

$$
\left|\partial^{m} f(x)-\partial^{m} f(y)\right| \leq C|x-y|^{s-m} .
$$

On the one hand, if $s<\gamma(0)$, then $f \in C_{l}^{s}\left(B\left(x_{0}, \delta\right)\right)$, for any $\delta<1 / 4$. Then, taking the limit when $\delta \rightarrow 0$, one has $\alpha_{l} \geq s$ for any $s<\gamma(0)$. Eventually, one concludes $\alpha_{l} \geq \gamma(0)$.

On the other hand, if $\alpha_{l}>\gamma(0)$, then there exists $s$ such that $\gamma(0)<s<\alpha_{l}$ and $\delta>0$ such that, for $\left|x-x_{0}\right|<\delta,\left|y-x_{0}\right|<\delta$,

$$
\left|\partial^{m} f(x)-\partial^{m} f(y)\right| \leq C|x-y|^{s-m} .
$$

Thus $f \in K_{x_{0}}^{s, 0}$, and $\gamma(0) \leq s$, which is absurd.

Proposition 2.4 Let $x_{0} \in \mathbb{R}$, and $f: \mathbb{R} \rightarrow \mathbb{R}$ such that $\gamma(0)>0$. The pointwise Hölder exponent of $f$ is the unique $t>0$ such that the frontier intersects the second diagonal $(t,-t)$, i.e. $\alpha_{p}$ is the unique $\alpha$ with $\alpha=\gamma(-\alpha)$.

Proof: The existence and the unicity of this intersection $\alpha$ is clear, since $\gamma(0)>0$ and since $\left|\gamma^{\prime}\left(s^{\prime}\right)\right|<1$ for $s^{\prime}>0$.

First, this intersection is located on the left of the pointwise exponent of $f$ at $x_{0}$ since we know that $f$ cannot belong to $K_{x_{0}}^{s, s^{\prime}}$ for $s>\alpha_{p}$. Thus $\alpha \leq \alpha_{p}$.

On the other hand, if we assume $\alpha<\alpha_{p}$, let us take $s$ such that $\alpha<s<\alpha_{p}$. By definition of $\alpha_{p}, f \in C_{x_{0}}^{s}$, and by Proposition 2.2, $f \in K_{x_{0}}^{s,-s}$, which is in contradiction with the unicity of $\alpha$.

Proposition 2.3 and 2.4 show that, as soon as $f$ has a minimal local regularity(i.e. $\gamma(0)>0)$, one can read the pointwise and local Hölder exponents from the frontier.

To end up with this section, let us notice that all the above propositions are only consequences of simple manipulations of the several exponents $s, s^{\prime}$, and $s+s^{\prime}$. In fact the definitions of $K_{x_{0}}^{s, s^{\prime}}$ spaces combine in a smart way two notions of regularity: the global regularity around $x_{0}$ and the pointwise regularity at $x_{0}$. They provide us with a deep understanding of the behavior of the considered function $f$ around $x_{0}$.

\section{Relation with 2-microlocal Spaces}

The main result of the paper is the following theorem, which identifies in the most interesting cases the 2-microlocal spaces $C_{x_{0}}^{s, s^{\prime}}$ with our spaces $K_{x_{0}}^{s, s^{\prime}}$. The previous propositions $(2.1)$ to (2.4), are in fact consequences of the following theorem 3.1, since the corresponding 
properties have been proved to hold for $C_{x_{0}}^{s, s^{\prime}}$ spaces. However, we have left them to show how easier propositions (2.3) and (2.4) are to prove in our frame than in the 2-microlocal frame.

\subsection{Main Result}

Theorem 3.1 Let $x_{0} \in \mathbb{R}$, and $s, s^{\prime}$ be two real numbers such that $s+s^{\prime}>0, s+s^{\prime} \notin \mathbb{N}$, and $s^{\prime}<0$. Then

$$
\left(f \in C_{x_{0}}^{s, s^{\prime}}\right) \Leftrightarrow\left(f \in K_{x_{0}}^{s, s^{\prime}}\right) .
$$

Let us say first a few words about the contraints on $s$ and $s^{\prime} . s>0$ implies that there exists a sort of Taylor expansion of $f$ at $x_{0}$ (because $f \in C_{x_{0}}^{s}$ ). As shown before, the condition $s+s^{\prime}>0$ implies a global regularity for the function in a neighborhood of $x_{0}$.

Theorem 3.1 does not contain the critical case $s+s^{\prime}=0$. Indeed, $C_{x_{0}}^{s,-s}$ contains distributions, which obviousy do not belong to any $K_{x_{0}}^{s,-s}$ spaces. $K_{x_{0}}^{s,-s}$ spaces are thus strictly included in $C_{x_{0}}^{s,-s}$ spaces.

Theorem 3.1 can be compared to Theorem 2.2. Indeed, Theorem 2.2 assumes a minimal global regularity for the considered function $f$ (namely $f \in C^{l o g}$ ) to estimate quantities of the type $\left|f(x)-P\left(x-x_{0}\right)\right|$, while Theorem 3.1 provides us with an equivalence and allows us to estimate differences of the type $|f(x)-f(y)|$, for any couple of points $(x, y)$ in a neighborhood of $x_{0}$. This gain of accuracy is due to the fact that we fully use the assumption of local regularity (i.e. $s+s^{\prime}>0$ ).

We focus on the case $0<s+s^{\prime}<1, s<1$, when the analysis is done using an orthonormal basis of compactly supported wavelets. This restriction is of great interest for practical purposes, as we shall see later. The proof of Theorem 3.1 in the general case is given in Section 6.

\subsection{Proof in a simple case}

Proof: We assume that $x_{0}=0$, and that $s^{\prime} \leq 0,0<s<1$ and $0<s+s^{\prime}<1$. The definition of $K_{x_{0}}^{s, s^{\prime}}$ spaces takes here a nice form, i.e.

$$
|f(x)-f(y)| \leq C|x-y|^{s+s^{\prime}}\left(|x-y|+\left|x-x_{0}\right|\right)^{-s^{\prime}} .
$$

The important case of functions which are continuous but nowhere differentiable is contained in this frame.

We prove Theorem 3.1 when an orthonormal wavelet basis generated with a compactly supported wavelet is used. This is slightly different and easier than the general case. For this, consider a Daubechies wavelet $\psi$, compactly supported, which is supposed to have at least 2 vanishing moments (see [4] for the existence and the construction of such a function). For each couple $(j, k)$, we denote by $S_{j, k}$ the support of $\psi_{j, k}$, the translated-dilated version 
of $\psi$. Namely, one has $\psi_{j, k}(x)=\psi\left(2^{j} x-k\right)$ and $S_{j, k}=\left[(k-K) 2^{-j},(k+K) 2^{-j}\right]$, where $2 K+1$ is the length of the support of $\psi$. The corresponding wavelet coefficient is

$$
d_{j, k}=2^{j} \int f(x) \psi_{j, k}(x) d x .
$$

To prove the result, we will use the characterization of the $C_{x_{0}}^{s, s^{\prime}}$ spaces by wavelet coefficients, recalled in Theorem 2.1.

\section{1. $K_{0}^{s, s^{\prime}} \subset C_{0}^{s, s^{\prime}}$}

Assume that $f \in K_{0}^{s, s^{\prime}}$. Then (17) holds. We want to study the wavelet coefficients $d_{j, k}$.

Using the first vanishing moment of the wavelet, we write

$$
\begin{aligned}
\left|d_{j, k}\right| & =\left|\int f(x) 2^{j} \psi_{j, k}(x) d x\right|=2^{j}\left|\int_{S_{j, k}}\left(f(x)-f\left(k 2^{-j}\right)\right) \psi_{j, k}(x) d x\right| \\
& =2^{j} \int_{S_{j, k}}\left|x-k 2^{-j}\right|^{s+s^{\prime}}\left(\left|x-k 2^{-j}\right|+\left|k 2^{-j}\right|\right)^{-s^{\prime}}\left|\psi_{j, k}(x)\right| d x
\end{aligned}
$$

On the interval $S_{j, k},\left|x-k 2^{-j}\right|$ is bounded by $K 2^{-j}$, where $K$ does not depend on $x, y$, $j$ or $k$. Then

$$
\left|x-k 2^{-j}\right|+\left|k 2^{-j}\right| \leq C 2^{-j}(1+|k|),
$$

thus $\left(\left|x-k 2^{-j}\right|+|x|\right)^{-s^{\prime}} \leq C 2^{j s^{\prime}}(1+|k|)^{-s^{\prime}}$. Moreover, on $S_{j, k}$, one also has $\left|x-k 2^{-j}\right|^{s+s^{\prime}} \leq$ $C 2^{-j\left(s+s^{\prime}\right)}$ on $S_{j, k}$. One deduces that

$$
\left|d_{j, k}\right| \leq C 2^{j s^{\prime}}(1+|k|)^{-s^{\prime}} 2^{-j\left(s+s^{\prime}\right)} \int_{S_{j, k}} 2^{j}\left|\psi_{j, k}(x)\right| d x \leq C 2^{-j s}(1+|k|)^{-s^{\prime}},
$$

since $\int 2^{j}\left|\psi_{j, k}(x)\right| d x$ is a constant. Thus, $f$ indeed belongs to $C_{0}^{s, s^{\prime}}$.

\section{2. $C_{0}^{s, s^{\prime}} \subset K_{0}^{s, s^{\prime}}$}

We suppose that the wavelet coefficients of $f$ verify $\left|d_{j, k}\right| \leq C 2^{-j s}(1+|k|)^{-s^{\prime}}\left(f \in C_{0}^{s, s^{\prime}}\right)$. We aim to show that $f$ satisfies (17).

Since $s+s^{\prime}>0, C_{0}^{s, s^{\prime}} \subset C^{s+s^{\prime}}$ around 0 , and we are allowed to use the reconstruction formula

$$
f(x)=\sum_{j} \sum_{k} d_{j, k} \psi_{j, k}(x)
$$

As explained before, it is enough to treat the case $|y| \leq x$. We have to to study the difference

$$
|f(x)-f(y)|=\left|\sum_{j} \sum_{k} d_{j, k}\left(\psi_{j, k}(x)-\psi_{j, k}(y)\right)\right| .
$$


We denote by $j_{0}$ be the integer such that

$$
2^{-j_{0}-1} \leq|x-y|<2^{-j_{0}} .
$$

The difference (19) can be splitted into three different expressions

$$
\begin{aligned}
|f(x)-f(y)| & \leq \sum_{j \leq j_{0}-1} \sum_{k}\left|d_{j, k}\right|\left|\psi_{j, k}(x)-\psi_{j, k}(y)\right| \\
& +\sum_{j \geq j_{0}} \sum_{k}\left|d_{j, k}\right|\left|\psi_{j, k}(x)\right| \\
& +\sum_{j \geq j_{0}} \sum_{k}\left|d_{j, k}\right|\left|\psi_{j, k}(y)\right|
\end{aligned}
$$

Let us study first the term $(I I)$.

$$
\sum_{j \geq j_{0}} \sum_{k}\left|d_{j, k}\right|\left|\psi_{j, k}(x)\right| \leq C \sum_{j \geq j_{0}} \sum_{k} 2^{-j s}(1+|k|)^{-s^{\prime}}\left|\psi_{j, k}(x)\right| .
$$

The crucial fact here is the following: if $x$ and $j$ are fixed, only a fixed number of $\psi_{j, k}(x)$ are different from 0 , namely $2 K+1$, i.e. the length of the support of $\psi$. This corresponds to the couples of indices $(j, k)$ such that $\left|x-k 2^{-j}\right| \leq K 2^{-j}$, i.e. $(1+|k|) \sim\left(1+2^{j}|x|\right)$.

Then, using that the dilated-translated $\psi_{j, k}$ 's are bounded by the same constant $M(M$ and $K$ are independant of $s, s^{\prime}, j$ and $k$ ), one has

$$
\sum_{j \geq j_{0}} \sum_{k}\left|d_{j, k}\right|\left|\psi_{j, k}(x)\right| \leq C \sum_{j \geq j_{0}}(2 K+1) M 2^{-j s}\left(1+2^{j}|x|\right)^{-s^{\prime}} .
$$

If $j \geq j_{0}, 2^{j}|x|>1 / 2$, thus $2^{j}|x|<2^{j}|x|+1 \leq 4.2^{j}|x|$. One thus has

$$
\begin{aligned}
\sum_{j \geq j_{0}} \sum_{k}\left|d_{j, k}\right|\left|\psi_{j, k}(x)\right| & \leq C \sum_{j \geq j_{0}} 2^{-j\left(s+s^{\prime}\right)}|x|^{-s^{\prime}} \leq C 2^{-j_{0}\left(s+s^{\prime}\right)}|x|^{-s^{\prime}} \\
& \leq C|x-y|^{s+s^{\prime}}|x|^{-s^{\prime}}
\end{aligned}
$$

since (20) holds. Then, using that $|x-y| \leq 2|x|$, the last inequality gives

$$
\sum_{j \geq j_{0}} \sum_{k}\left|d_{j, k}\right|\left|\psi_{j, k}(x)\right| \leq C|x-y|^{s+s^{\prime}}(|x-y|+|x|)^{-s^{\prime}}
$$

which is the correct bound.

The third term is bounded by the same method as described above.

We now move to the first term, which is a little bit more delicate to study. We will use the derivative of the wavelet $\psi$. Indeed, one remarks that

$$
\left|\psi_{j, k}(x)-\psi_{j, k}(y)\right| \leq|x-y| \sup _{z \in[y, x]}\left|\psi_{j, k}^{\prime}(z)\right| .
$$


But we know that $\psi_{j, k}^{\prime}(x)=\left(\psi\left(2^{j} x-k\right)\right)^{\prime}=2^{j} \psi^{\prime}\left(2^{j} x-k\right)$, which is uniformly bounded by $C 2^{j}$. Thus one has the property

$$
\left|\psi_{j, k}(x)-\psi_{j, k}(y)\right| \leq C|x-y| 2^{j} .
$$

The sum in $k$ contains only a fixed number $2 K+1$ of non-zero terms ( $K$ is still independant of $x$ and $y$ ), and for these $k$ 's, by the same arguments as before, $(1+|k|)^{-s^{\prime}} \sim\left(1+2^{j}|x|\right)^{-s^{\prime}}$. Using (22), one writes

$$
\begin{aligned}
(I) & \leq C \sum_{j \leq j_{0}-1} \sum_{k}\left|d_{j, k}\right| 2^{j}|x-y| \leq C|x-y| \sum_{j \leq j_{0}-1}(2 K+1) 2^{-j s}(1+|k|)^{-s^{\prime}} \\
& \leq C|x-y| \sum_{j \leq j_{0}-1} 2^{j(1-s)}\left(1+2^{j}|x|\right)^{-s^{\prime}}
\end{aligned}
$$

Moreover, $\left(1+2^{j}|x|\right)^{-s^{\prime}} \leq C 1+\left(2^{j}|x|\right)^{-s^{\prime}}$, and

$$
\begin{aligned}
(I) & \leq C|x-y| 2^{j_{0}(1-s)}+2^{j_{0}\left(1-\left(s+s^{\prime}\right)\right)}|x|^{-s^{\prime}} \\
& \leq C|x-y|\left(|x-y|^{s-1}+|x-y|^{s+s^{\prime}-1}|x|^{-s^{\prime}}\right) \\
& \leq C|x-y|^{s+s^{\prime}}\left(|x-y|^{-s^{\prime}}+|x|^{-s^{\prime}}\right) \leq C|x-y|^{s+s^{\prime}}(|x-y|+|x|)^{-s^{\prime}}
\end{aligned}
$$

The key at this point was that $1-\left(s+s^{\prime}\right)$ is strictly positive by construction. One now upper-bounds $|x|^{-s^{\prime}}$ by $(|x-y|+|x|)^{-s^{\prime}}$.

This ends the proof of Theorem 3.1 in the simple case.

\subsection{Applications}

We give here two applications of Theorem 3.1. We first exhibit some classes of functions that will belong to $C_{x_{0}}^{s, s^{\prime}}$. Second we give a decomposition of any function of $C_{x_{0}}^{s, s^{\prime}}$ into "simpler" functions. These propositions were already proved in a more general frame in [11], but our approach shows how easier they are to prove with the help of the $K_{x_{0}}^{s, s^{\prime}}$ characterization.

Proposition 3.1 Let us assume that $s^{\prime}<0$ and $s+s^{\prime}>0$. Let $\left\{U_{j}(x)\right\}_{j \in \mathbb{N}}$ be a sequence of functions that satisfy, for every $|\alpha| \leq N$,

$$
\left|\partial^{\alpha} U_{j}(x)\right| \leq C(1+|x|)^{-s^{\prime}} .
$$

Then the function $f$ defined by

$$
f(x)=\sum_{j=0}^{+\infty} 2^{-s j} U_{j}\left(2^{j}\left(x-x_{0}\right)\right)
$$

belongs to $C_{x_{0}}^{s, s^{\prime}}$. 
Proof: We give here the proof of this proposition in the case where $s+s^{\prime}<1, s<1$, the general case only needs an easy adaptation of the following.

Let us take $f$ defined by (24), and let us now study the differences $|f(x)-f(y)|$. Let $j_{0}$ be the integer such that $2^{-j_{0}} \leq|x-y| \leq 2^{-j_{0}-1}$. Then,

$$
\begin{aligned}
|f(x)-f(y)|= & \left|\sum_{j=0}^{+\infty} 2^{-s j}\left(U_{j}\left(2^{j}\left(x-x_{0}\right)\right)-U_{j}\left(2^{j}\left(y-x_{0}\right)\right)\right)\right| \\
\leq & \sum_{j=0}^{j_{0}} 2^{-s j}\left|U_{j}\left(2^{j}\left(x-x_{0}\right)\right)-U_{j}\left(2^{j}\left(y-x_{0}\right)\right)\right| \\
& +\sum_{j=j_{0}+1}^{+\infty} 2^{-s j}\left|U_{j}\left(2^{j}\left(x-x_{0}\right)\right)-U_{j}\left(2^{j}\left(y-x_{0}\right)\right)\right|
\end{aligned}
$$

When $j \geq j_{0}+1$, by (23), one obtains

$$
\left|U_{j}\left(2^{j}\left(x-x_{0}\right)\right)-U_{j}\left(2^{j}\left(y-x_{0}\right)\right)\right| \leq 2\left|U_{j}\left(2^{j}\left(x-x_{0}\right)\right)\right| \leq C\left(1+2^{j}\left|x-x_{0}\right|\right)^{-s^{\prime}},
$$

and thus

$$
\begin{aligned}
(I I) & \leq C \sum_{j=j_{0}+1}^{+\infty} 2^{-s j}\left(1+2^{j}\left|x-x_{0}\right|\right)^{-s^{\prime}} \leq C \sum_{j=j_{0}+1}^{+\infty} 2^{-s j}+2^{-\left(s+s^{\prime}\right) j}\left|x-x_{0}\right|^{-s^{\prime}} \\
& \leq C 2^{-s j_{0}}+2^{-\left(s+s^{\prime}\right) j_{0}}\left|x-x_{0}\right|^{-s^{\prime}} \leq C|x-y|^{s}
\end{aligned}
$$

since $2^{-j_{0}} \sim\left|x-x_{0}\right|$.

Consider now the other term $(I)$.

$$
\begin{aligned}
(I) & \leq C \sum_{j=0}^{j_{0}} 2^{-s j}\left|2^{j}\left(x-x_{0}\right)-2^{j}\left(y-x_{0}\right)\right| \sup _{z \in\left[2^{j}\left(x-x_{0}\right), 2^{j}\left(y-x_{0}\right)\right]}\left|\partial^{1} U_{j}(z)\right| \\
& \leq C \sum_{j=0}^{j_{0}} 2^{-s j} 2^{j}|x-y| \max \left(\left(1+2^{j}\left|x-x_{0}\right|\right)^{-s^{\prime}},\left(1+2^{j}\left|y-x_{0}\right|\right)^{-s^{\prime}}\right) \\
& \leq C 2^{(1-s) j_{0}}\left(1+2^{j_{0}}\left|x-x_{0}\right|\right)^{-s^{\prime}}|x-y| \\
& \leq C|x-y|^{s}\left(1+\frac{\left|x-x_{0}\right|}{|x-y|}\right)^{-s^{\prime}} \leq C|x-y|^{s+s^{\prime}}\left(|x-y|+\left|x-x_{0}\right|\right)^{-s^{\prime}}
\end{aligned}
$$

where one has assumed that $\left(1+2^{j}\left|x-x_{0}\right|\right)^{-s^{\prime}} \geq\left(1+2^{j}\left|y-x_{0}\right|\right)^{-s^{\prime}}$.

The following proposition gives a decomposition of any function $f \in K_{x_{0}}^{s, s^{\prime}}$ into two terms of different behaviors, the first one being regular and the second one containing the "oscillatory" behavior of $f$ around $x_{0}$. It has already been proved in a more general case by Y. Meyer in [11].

$\mathrm{RR} \mathrm{n}^{\circ} 4545$ 
Proposition 3.2 Let $s, s^{\prime}$ be two real numbers such that $s+s^{\prime}>0, s^{\prime}<0$, and $x_{0} \in \mathbb{R}$. Then the following propositions are equivalent

- $f \in K_{x_{0}}^{s, s^{\prime}}$

- there exist a constant $\delta>0$, a polynomial $P$ of degree smaller than [s], and a function $h$ which satisfies $h \in C^{s+s^{\prime}}\left(\left[x_{0}-\delta, x_{0}+\delta\right]\right)$ and $|h(x)| \leq C\left|x-x_{0}\right|^{s+s^{\prime}}$, such that

$$
f(x)=P(x)+\left|x-x_{0}\right|^{-s^{\prime}} h(x) .
$$

Proof: We treat the case $0<s+s^{\prime}<1$ and $x_{0}=0$.

Assume $f \in K_{0}^{s, s^{\prime}}$, and $|y| \leq x$. There exists a polynomial $P$ of degree smaller than $s$ such that (9) holds. Let us define the functions $g$ and $h$ by

$$
g(x)=\frac{f(x)-P(x)}{|x|^{[s]}}, \quad h(x)=\frac{f(x)-P(x)-g(0) x^{[s]}}{|x|^{-s^{\prime}}} .
$$

One knows that, for all $x, y$ close enough to 0 ,

$$
|g(x)-g(y)| \leq C|x-y|^{s+s^{\prime}}(|x-y|+|x|)^{-s^{\prime}-[s]},
$$

and that $h(x)=|x|^{s^{\prime}+[s]}(g(x)-g(0))$. One always has $-1<s^{\prime}+[s]<1$. If $s^{\prime}+[s]=0$, the result is obvious, thus we restrict the study to $s^{\prime}+[s] \neq 0$.

First note that if $y=0$,

$$
|h(x)-h(y)|=|h(x)|=|x|^{s^{\prime}+[s]}|g(x)-g(0)| \leq C|x|^{s^{\prime}+[s]}|x|^{s-[s]}=C|x|^{s+s^{\prime}} .
$$

Now one can assume that $x \neq 0$ and $y \neq 0$, and thus, denoting $\tilde{g}(x)=g(x)-g(0)$,

$$
|h(x)-h(y)| \leq\left.|| x\right|^{s^{\prime}+[s]} \tilde{g}(x)-|y|^{s^{\prime}+[s]} \tilde{g}(y) \mid
$$

Using that $|\tilde{g}(x)| \leq C|x|^{s-[s]}$ for all $x$ close enough to 0 and (9), one obtains

$$
\begin{aligned}
|h(x)-h(y)| \leq & |x|^{s^{\prime}+[s]}|\tilde{g}(x)-\tilde{g}(y)|+\left.|| x\right|^{s^{\prime}+[s]} \tilde{g}(y)-|y|^{s^{\prime}+[s]} \tilde{g}(y) \mid \\
\leq & |x|^{s^{\prime}+[s]}|\tilde{g}(x)-\tilde{g}(y)|+\left.|\tilde{g}(y)||| x\right|^{s^{\prime}+[s]}-|y|^{s^{\prime}+[s]} \mid \\
\leq & C\left(|x|^{s^{\prime}+[s]}|x-y|^{s+s^{\prime}}(|x-y|+|x|)^{-s^{\prime}-[s]}\right. \\
& +\left.|g(y)||| x\right|^{s^{\prime}+[s]}-|y|^{s^{\prime}+[s]} \mid
\end{aligned}
$$

Then we make the same kind of manipulations as before. For the first term, one uses $|x| \leq|x-y|+|x| \leq 3|x|$ to get

$$
\begin{aligned}
|x-y|^{s+s^{\prime}}|x|^{s^{\prime}+[s]}(|x-y|+|x|)^{-s^{\prime}-[s]} & \leq C|x-y|^{s+s^{\prime}}|x|^{s^{\prime}+[s]}|x|^{-s^{\prime}-[s]} \\
& \leq C|x-y|^{s+s^{\prime}} .
\end{aligned}
$$

The second term is more delicate to study: 
- if $|x-y| \leq|y|$, then

$$
\begin{aligned}
\left.|\tilde{g}(y)||| x\right|^{s^{\prime}+[s]}-|y|^{s^{\prime}+[s]} \mid & \leq C|y|^{s-[s]}|x-y||y|^{s^{\prime}+[s]-1} \\
& \leq C|x-y|^{s+s^{\prime}}\left(\frac{|x-y|}{|y|}\right)^{1-\left(s+s^{\prime}\right)} \leq C|x-y|^{s+s^{\prime}} .
\end{aligned}
$$

- if $|y| \leq|x-y|$ (i.e. $y<x / 2$ ), two cases must be separated

- if $-1<s^{\prime}+[s]<0,\left.|| x\right|^{s^{\prime}+[s]}-\left.|y|^{s^{\prime}+[s]}|\leq C| y\right|^{s^{\prime}+[s]}$, and

$$
\left.|\tilde{g}(y)||| x\right|^{s^{\prime}+[s]}-\left.|y|^{s^{\prime}+[s]}|\leq C| y\right|^{s-[s]}|y|^{s^{\prime}+[s]} \leq C|y|^{s+s^{\prime}} \leq C|x-y|^{s+s^{\prime}} .
$$

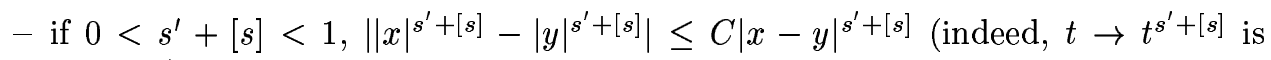
concave), and

$$
\begin{aligned}
\left.|\tilde{g}(y)||| x\right|^{s^{\prime}+[s]}-|y|^{s^{\prime}+[s]} \mid & \leq C|y|^{s-[s]}|x-y|^{s^{\prime}+[s]} \\
& \leq C|x-y|^{s-[s]}|x-y|^{s^{\prime}+[s]} \leq C|x-y|^{s+s^{\prime}} .
\end{aligned}
$$

The function $h$ belongs to $C^{s+s^{\prime}}\left(\left[x_{0}-\delta, x_{0}+\delta\right]\right)$.

Let us assume now that $f$ satisfies (25) for a certain polynomial $P$ and a function $h$, but does not satisfy (9).

Since (9) is not verified, one can find two sequences of real numbers $\left\{x_{n}\right\}_{n}$ and $\left\{y_{n}\right\}_{n}$, such that, for all $n$,

$$
\left|g\left(x_{n}\right)-g\left(y_{n}\right)\right| \geq n\left|x_{n}-y_{n}\right|^{s+s^{\prime}}\left(\left|x_{n}-y_{n}\right|+\left|x_{n}\right|\right)^{-s^{\prime}-[s]} .
$$

Since all the properties are local, around $x_{0}$, one can extract from these sequences two subsequences (still denoted by $x_{n}$ and $y_{n}$ ) that will satisfy $\lim _{n} x_{n}=X$ and $\lim _{n} y_{n}=Y$, and

$$
\left|g\left(x_{n}\right)-g\left(y_{n}\right)\right| \geq C_{n}\left|x_{n}-y_{n}\right|^{s+s^{\prime}}\left(\left|x_{n}-y_{n}\right|+\left|x_{n}\right|\right)^{-s^{\prime}-[s]},
$$

with $C_{n} \rightarrow+\infty$ when $n \rightarrow+\infty$.

First case $X \neq Y, X \neq 0$ and $Y \neq 0$. Since $g$ is continuous, using (27) and the fact that $\left|x_{n}-y_{n}\right| \rightarrow_{n}|X-Y|$ and $\left|x_{n}\right| \rightarrow_{n}|X|$, one obtains that $\forall n$ large enough,

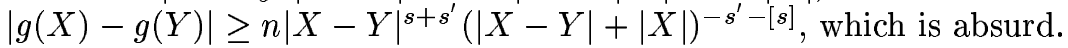

Second case $X=0$ and $Y \neq 0$. This case is treated similarly as the preceding one.

Third case $X=Y=0$. One can assume that, for all $n,\left|x_{n}\right| \geq\left|y_{n}\right|$.

By definition one has, for all $x,|g(x)| \leq C|x|^{s-[s]}$. Thus, for all $n,\left|g\left(x_{n}\right)-g\left(y_{n}\right)\right| \leq$ $C\left(\left|x_{n}\right|^{s-[s]}+\left|y_{n}\right|^{s-[s]}\right) \leq 2 C\left|x_{n}\right|^{s-[s]}$. On the other hand, using (27), one has

$$
\begin{aligned}
2 C\left|x_{n}\right|^{s-[s]} & \geq\left|g\left(x_{n}\right)-g\left(y_{n}\right)\right| \geq C_{n}\left|x_{n}-y_{n}\right|^{s+s^{\prime}}\left(\left|x_{n}-y_{n}\right|+\left|x_{n}\right|\right)^{-s^{\prime}-[s]} \\
& \geq C_{n}\left|x_{n}-y_{n}\right|^{s+s^{\prime}}\left|x_{n}\right|^{-s^{\prime}-[s]}
\end{aligned}
$$


The lower-bound $\left(\left|x_{n}-y_{n}\right|+\left|x_{n}\right|\right)^{-s^{\prime}-[s]}$ by $\left|x_{n}\right|^{-s^{\prime}-[s]}$ holds even when $-s^{\prime}-[s]<0$, still because $\left|x_{n}\right| \sim\left(\left|x_{n}-y_{n}\right|+\left|x_{n}\right|\right)$ for all $n$. One thus obtains $\left|x_{n}\right| \geq C_{n}^{\frac{1}{s+s}}\left|x_{n}-y_{n}\right|$, which can be rewritten as

$$
\left|x_{n}-y_{n}\right|=o\left(\left|x_{n}\right|\right)
$$

(28) says that the couples of points where the inequality (25) may fail must satisfy some strong properties: they converge together to 0 , and the differences $\left|x_{n}-y_{n}\right|$ are small while the differences $\left|g\left(x_{n}\right)-g\left(y_{n}\right)\right|$ stay large. Intuitively it corresponds to the case of strong oscillations around 0 .

Let us show that this is impossible. One would have

$$
\left|y_{n}\right|^{-s^{\prime}-[s]}=\left|x_{n}\right|^{-s^{\prime}-[s]}+\left(-s^{\prime}\right)\left|x_{n}-y_{n}\right|\left|z_{n}\right|^{-s^{\prime}-[s]-1},
$$

where $z_{n}$ is a real number between $x_{n}$ and $y_{n}$. Then,

$$
\begin{aligned}
\left|g\left(x_{n}\right)-g\left(y_{n}\right)\right|= & \left.|| x_{n}\right|^{-s^{\prime}-[s]} h\left(x_{n}\right)-\left|y_{n}\right|^{-s^{\prime}-[s]} h\left(y_{n}\right) \mid \\
\leq & \left|x_{n}\right|^{-s^{\prime}-[s]}\left|h\left(x_{n}\right)-h\left(y_{n}\right)\right| \\
& +C\left|h\left(y_{n}\right)\right|\left|x_{n}-y_{n}\right|\left|z_{n}\right|^{-s^{\prime}-[s]-1} \\
\leq & C\left|x_{n}\right|^{-s^{\prime}-[s]}\left|x_{n}-y_{n}\right|^{s+s^{\prime}} \\
& +C\left|y_{n}\right|^{s+s^{\prime}}\left|x_{n}-y_{n}\right|\left|z_{n}\right|^{-s^{\prime}-[s]-1}
\end{aligned}
$$

since $h \in C^{s+s^{\prime}}(\mathbb{R})$.

The first term in the last inequality is bounded by $\left|x_{n}-y_{n}\right|^{s+s^{\prime}}\left(\left|x_{n}-y_{n}\right|+\left|x_{n}\right|\right)^{-s^{\prime}-[s]}$. Let us deal with the last term. Using that $\left|z_{n}\right| \sim\left|x_{n}\right| \sim\left|y_{n}\right|$, one verifies that

$$
\begin{aligned}
\left|y_{n}\right|^{s+s^{\prime}}\left|x_{n}-y_{n}\right|\left|z_{n}\right|^{-s^{\prime}-[s]-1} & \leq C\left|y_{n}\right|^{s+s^{\prime}}\left|x_{n}-y_{n}\right|\left|y_{n}\right|^{-s^{\prime}-[s]-1} \\
& \leq C\left|x_{n}-y_{n}\right|\left|y_{n}\right|^{s-[s]-1} \\
& \leq C\left|x_{n}-y_{n}\right|^{s+s^{\prime}}\left|x_{n}-y_{n}\right|^{1-\left(s+s^{\prime}\right)}\left|y_{n}\right|^{s-[s]-1} \\
& \leq C\left|x_{n}-y_{n}\right|^{s+s^{\prime}}\left(\left|x_{n}-y_{n}\right|+\left|y_{n}\right|\right)^{-s^{\prime}-[s]}
\end{aligned}
$$

This eventually gives

$$
\left|g\left(x_{n}\right)-g\left(y_{n}\right)\right| \leq C\left|x_{n}-y_{n}\right|^{s+s^{\prime}}\left(\left|x_{n}-y_{n}\right|+\left|y_{n}\right|\right)^{-s^{\prime}-[s]},
$$

in contradiction with (27).

The main interest of the last proofs is to show that it is possible to check all the properties of the functions belonging to $C_{x_{0}}^{s, s^{\prime}}$ spaces (with $s+s^{\prime} \geq 0$ ) using only elementary arguments and a time domain analysis. 


\section{Algorithms}

\subsection{Background Ideas}

There are three major justifications for the use of $K_{x_{0}}^{s, s^{\prime}}$ spaces for the characterization of regularity in practical applications.

- $K_{x_{0}}^{s, s^{\prime}}$ spaces give a rather rich description of the regularity structure.

- The computation of both exponents $\left(s, s^{\prime}\right)$ is performed using directly the values of the function. One does not lose information by integrating or smoothing the data. Moreover one can extract from the frontier the usual information, i.e. the Hölder exponents, with a better accuracy since one uses for that many other computations.

- it may seem harder to estimate a frontier of a domain in $\mathbb{R}^{2}$ than only one regularity exponent. But this is not the case. The main reason is that we are using more information: in fact, using (9) we extract the whole information avalaible in the data. Combined with the fact that a frontier must satisfy a number of constraints such that its general aspect is known, this leads to some estimation procedures.

The formula defining the spaces $K_{x_{0}}^{s, s^{\prime}}$ for $s>1$ involves a polynomial which approximates the data (a kind of Taylor expansion), which is accessible only with the help of finite differences. This makes harder the implementation of an algorithm in these situations. We then focus on the simpler case $s<1$ and $s+s^{\prime}<1$, where we have already seen that there is no polynomial in the definition of $K_{x_{0}}^{s, s^{\prime}}$. An algorithm for estimating this part of the frontier has been proposed in [9]. We describe here another approach.

\subsection{Implementation}

We want to estimate the $K_{x_{0}}^{s, s^{\prime}}$-frontier of a function $f$ at a point $x_{0}$. We start with formula (17)

$$
|f(x)-f(y)| \leq C|x-y|^{s+s^{\prime}}\left(|x-y|+\left|x-x_{0}\right|\right)^{-s^{\prime}} .
$$

We assume that we have at our disposal the discrete values $\left\{f_{i}\right\}_{i=1, \ldots, N}$ of a function $f$ at the points $\left\{x_{i}\right\}_{i=1, \ldots, N}$ (note that we do not need to assume that the $\left\{x_{i}\right\}_{i=1, \ldots, N}$ are equidistant). If $f \in K_{x_{0}}^{s, s^{\prime}}, \forall i, j$,

$$
\left|f_{i}-f_{j}\right| \leq C\left|x_{i}-x_{j}\right|^{s+s^{\prime}}\left(\left|x_{i}-x_{j}\right|+\left|x_{i}-x_{0}\right|\right)^{-s^{\prime}} .
$$

Define $x_{i, j}$ by $x_{i, j}=\log \left(\left|x_{i}-x_{j}\right|\right)$ and $y_{i, j, s^{\prime}}$ by

$$
y_{i, j, s^{\prime}}=\log \left(\left|f_{i}-f_{j}\right|\right)+s^{\prime} \log \left(1+\frac{\left|x_{i}-x_{0}\right|}{\left|x_{i}-x_{j}\right|}\right) .
$$

Then, (29) reads $\forall \lambda=(i, j), y_{\lambda, s^{\prime}} \leq C x_{\lambda}$. 
Now fix an exponent $s^{\prime}$. In order to obtain the other exponent $s$ as a function of $s^{\prime}$, it suffices to make a regression on the maxima of the set of couples $\left(x_{\lambda}, y_{\lambda}\right)$ (where $\left.\lambda \in[1, \ldots, N]^{2}\right)$ to find the corresponding exponent $s=\Gamma\left(s^{\prime}\right)$.

The practical implementation follows the rules

- Choose a set of $n$ discretised values of $s^{\prime},\left\{s_{1}^{\prime}, s_{2}^{\prime}, \ldots, s_{n}^{\prime}\right\}$, ranging typically in $[-1,0.5]$.

- For each $s_{i}^{\prime}$, compute the corresponding $y_{\lambda, s_{i}^{\prime}}$ and $x_{\lambda}$.

- Find the largest $y_{\lambda, s_{i}^{\prime}}$, when $\lambda$ belongs to $\Lambda_{x_{0}}=\left\{(k, l) /\left|x_{0}-x_{k}\right|<1 / 4\right.$ and $\left|x_{0}-x_{l}\right|<$ $1 / 4\}$. We obtain the $y_{\mu, s_{i}^{\prime}}$, where $\mu$ belongs to a subset of $\Lambda_{x_{0}}$.

- Make a linear regression on the set of couples $\left(x_{\mu, s^{\prime}}, y_{\mu, s^{\prime}}\right)$.

- Then the slope of the straight line obtained by regression is the approximation of the exponent $s$ corresponding to the $s^{\prime}$.

A set of $n$ samples in the frontier of exponents $s=\Gamma\left(s^{\prime}\right)$ is eventually obtained. By a simple method of convexification, it can be modified into a convex set of samples. Applying this method we obtain an approximation of the frontier, which satisfies its basic theoretical properties: it is convex, non-decreasing, with a derivative with modulus less than 1.

A last but important remark is the following: since we use the simple version of the $K_{x_{0}}^{s, s^{\prime}}$ spaces (i.e. without polynomials), the formula we use can only be applied in the triangle $0<s<1, s^{\prime}<0$ and $0<s+s^{\prime}<1$. This implies that, for a function $f$ whose pointwise Hölder exponent is $\alpha<1$, the algorithm can not detect any regularity larger than $s=\alpha$, even when $s+s^{\prime}<0$. This equivalently means that the frontier the algorithm tries to estimate can not intersect the half-plane $\left\{\left(s, s^{\prime}\right) / s>\alpha\right\}$. This provides us with a sharp localization of the pointwise Hölder exponent.

\section{$5 \quad$ Numerical Results}

We present the results of the algorithm implemented in different cases: first the case of an isolated singularity, with three examples: a cusp singularity, a chirp singularity, and a sum of two chirps at the same point; then the more complicated cases of functions which are everywhere continuous, but nowhere differentiable: the Weierstrass function, the fractionnal Brownian motion, and the generalized Weierstrass function.

In all the figures, we plot the frontier found by the algorithm, and compare it with the theoretical one. We also plot the straight lines $s+s^{\prime}=0$, and $s+s^{\prime}=1$, which bound the validity of the results (indeed, remember that the formula we are using is only valid for $0<s+s^{\prime}<1$ and $s^{\prime}<0$ ). All the results were obtained using functions sampled on 1024 points. 

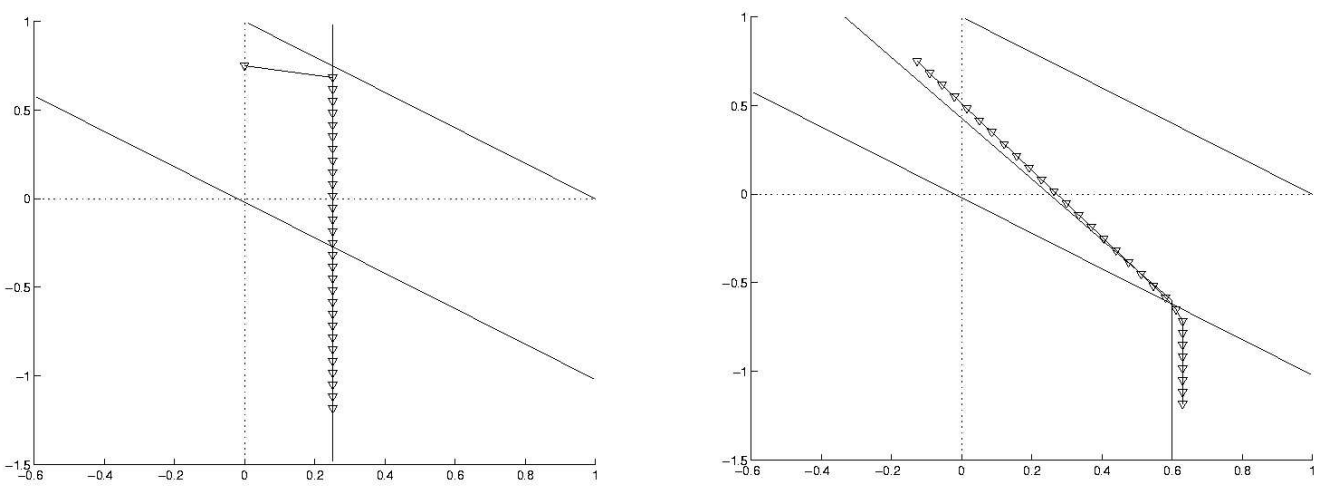

Figure 2: Left: Estimation of the frontier of the cusp $|x|^{0.25}$ at 0 . The estimation is plotted with triangles, the theoretical result is the straight line. Right: Estimation of the frontier of the chirp $|x|^{0.6} \sin \left(\frac{1}{|x|^{1.4}}\right)$ at 0 .

\subsection{A cusp}

The function considered here is $x \rightarrow|x|^{0.25}$. Since there is no oscillation phenomenon, the local and the pointwise Hölder exponents are both equal to $\alpha=0.25$. The theoretical frontier is a vertical line.

The estimation found for the common value of the two regularity exponents is 0.252 , which is extremely precise (see Figure 2).

\section{$5.2 \quad$ A chirp}

The function we study here is the chirp function, $|x|^{0.6} \sin \left(\frac{1}{\left.x\right|^{1.4}}\right)$. The theoretical Hölder exponents are $\alpha_{p}=0.6$, and $\alpha_{l}=\frac{0.6}{1+1.4}=0.25$.

The frontier computed by the algorithm and plotted in Figure 2 provides us with the estimations 0.27 and 0.62 for, respectively, the local and the pointwise exponent of $f$. One notices one more time the precision of the results, and also that the theoretical shape of the frontier is respected.

\subsection{A sum of two chirps}

The case of the sum of two chirps located at the same point is delicate. Indeed, it is very hard to distinguish the two behaviors, since there are two types of oscillations (at different frequencies).

We see that, in Figure 3, the two behaviors are identified, since, for example, the theorical pointwise and local exponents (respectively 0.5 and 0.25 ) are found with a good precision. 

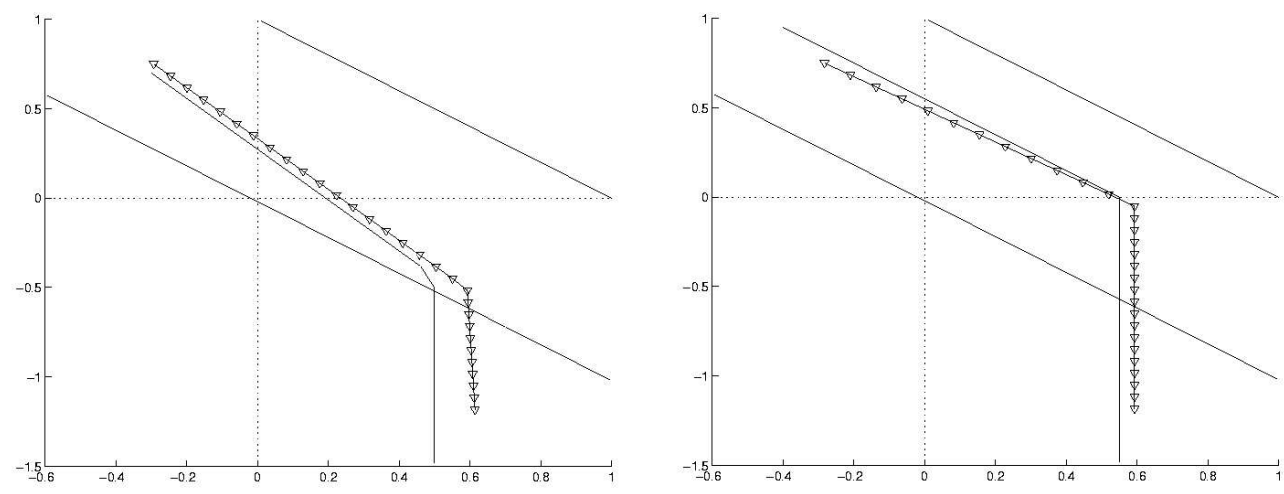

Figure 3: Left: Estimation of the frontier of the function $|x|^{0.8} \sin \left(\frac{1}{x^{3}}\right)+|x|^{0.5} \sin \left(\frac{1}{x^{0.25}}\right)$ at 0. Right: Estimation of the frontier of the Weierstrass function with $\lambda=3.23$ and $H=0.55$ at an arbitrary point.

The transition phase is not respected as it should be, but, away from it, the frontier is correctly approximated.

\subsection{A Weierstrass function}

The Weierstrass function, defined by

$$
W_{H}(x)=\sum_{j=0}^{+\infty} \lambda^{-j H} \sin \left(\lambda^{j} x\right),
$$

where $\lambda \geq 2$ and $0<H<1$, belongs to a more complicated type of functions. Indeed, it is well known that $W_{H}$ is everywhere continuous, nowhere differentiable, and that it has a Hölder exponent equal to $H$ at all points (see for example the original article [13] and [6]).

Remark that, in Figure 3, the knee of the frontier located around the axis $s^{\prime}=0$ is found and respected by the algorithm.

\subsection{An fBm}

A path of a Fractional Brownian Motion is another way of obtaining a signal for which the pointwise Hölder function is controlled, and is almost surely everywhere equal to a given exponent $H$ (see [1] for example).

We have tested the algorithm on an $\mathrm{fBm}$ with $H=0.7$ (see Figure 4). 

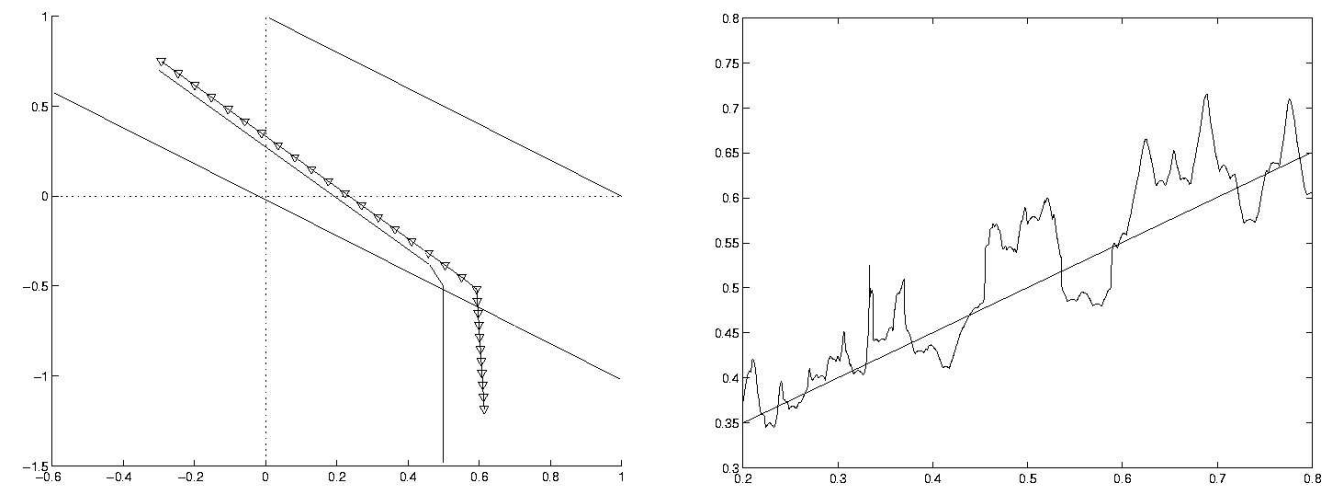

Figure 4: Left: Estimation of the frontier at an arbitrary point of an fBm with $H=0.7$. The knee in the frontier (at $s^{\prime}=0$ ) is still recovered by the algorithm, but it is smoother in the estimation than in the theoretical frontier. Right: Estimation of the pointwise Hölder exponents of a generalized Weierstrass function satisfying $\alpha_{p}(t)=t / 2+1 / 4$, from $t=0.2$ to $t=0.8$. The straight line is the theoretical pointwise Hölder function.

\subsection{A generalized Weierstrass function}

With the generalized Weierstrass functions, one can also control the regularity exponents, the difference with the classical Weierstrass functions being that one can now prescribe time varying pointwise exponents. We refer the reader to [3] for more details on this subject.

We have run the algorithm on a function generated with the contraint $\alpha_{p}(t)=t / 2+1 / 4$, and we plot the approximation of the pointwise exponents for the range $t \in[0.2,0.8]$ (thus the pointwise exponents are supposed to range in $[0.3,0.65]$ ). We present the result in Figure 4 .

\section{Proof of Theorem 3.1}

For proving this theorem, we use the wavelet based characterization of the space $C_{x_{0}}^{s, s^{\prime}}$. To simplify the notations, we will assume $x_{0}=0$.

Let $r$ and $N$ be two integers such that $r+\left(s+s^{\prime}\right)>2$ and $N>s+1$.

Let $\psi$ be a wavelet satisfying the following conditions:

$$
\begin{gathered}
\left|\partial^{\alpha} \psi(x)\right| \leq C_{q}(1+|x|)^{-q}, \text { if }|\alpha| \leq r \text { and } q \in \mathbb{N}, \\
\int x^{\beta} \psi(x) d x=0 \text { if }|\beta| \leq N-1 .
\end{gathered}
$$


These properties can be viewed as an efficient localization around 0 of the wavelet $\psi$ and its $r$ first derivatives. Then one has the equivalence between the two following properties [11]

$$
\begin{gathered}
f \in C_{0}^{s, s^{\prime}}, \\
\forall j \in \mathbb{N}, k \in \mathbb{Z},\left|d_{j, k}\right| \leq C 2^{-j s}\left(1+\left|k-2^{j} x_{0}\right|\right)^{-s^{\prime}} .
\end{gathered}
$$

where $d_{j, k}=\int f(x) 2^{j} \psi\left(2^{j} x-k\right) d x$ is the wavelet coefficient of $f$ at scale $j$ in $k$.

1. $K_{0}^{s, s^{\prime}} \subset C_{0}^{s, s^{\prime}}$

Let $f$ be a function in $K_{0}^{s, s^{\prime}}$. We treat the case $0<s+s^{\prime}<1$, the general case follows by replacing $\psi_{j, k}$ by $\psi_{j, k}^{(-m)}$ (a primitive of order $m$ of $\psi_{j, k}$, which can be chosen satisfying the conditions to be a wavelet. Thus the result will hold with a different wavelet basis).

We assume $k \geq 0$, without loss of generality. One can write (the integral is taken between $-\infty$ and $+\infty)$

$$
\begin{aligned}
\left|d_{j, k}\right| & =\left|\int f(x) 2^{j} \psi\left(2^{j} x-k\right) d x\right| \\
& =\left.2^{j}\left|\int\right| x\right|^{[s]}\left[\frac{f(x)-P(x)}{|x|^{[s]}}-\frac{f\left(k 2^{-j}\right)-P\left(k 2^{-j}\right)}{\left|k 2^{-j}\right|^{[s]}}\right] \psi\left(2^{j} x-k\right) d x \mid,
\end{aligned}
$$

for any polynomial $P$ of order smaller than $[s]$, because the wavelet has $N \geq[s]$ vanishing moments. Then, taking for $P$ the polynomial that appears in the definition of $K_{0}^{s, s^{\prime}}$ given by (9), one obtains

$$
\begin{aligned}
\left|d_{j, k}\right| & \leq C 2^{j} \int|x|^{[s]}\left|x-k 2^{-j}\right|^{s+s^{\prime}}\left(\left|x-k 2^{-j}\right|+\left|k 2^{-j}\right|\right)^{-s^{\prime}-[s]}\left|\psi\left(2^{j} x-k\right)\right| d x \\
& \leq C 2^{j} \int\left|x-k 2^{-j}\right|^{s+s^{\prime}}\left(\left|x-k 2^{-j}\right|+\left|k 2^{-j}\right|\right)^{-s^{\prime}}\left|\psi\left(2^{j} x-k\right)\right| d x,
\end{aligned}
$$

since one obviously has $|x|^{[s]} \leq\left(\left|x-k 2^{-j}\right|+\left|k 2^{-j}\right|\right)^{[s]}$. Then, using the localization of the wavelet $\psi$ for some $q$ that can be chosen very large,

$$
\begin{aligned}
\left|d_{j, k}\right| & \leq C 2^{j} \int\left|x-k 2^{-j}\right|^{s+s^{\prime}} \frac{\left(\left|x-k 2^{-j}\right|+\left|k 2^{-j}\right|\right)^{-s^{\prime}}}{\left(1+\left|2^{j} x-k\right|\right)^{q}} d x \\
& \leq C 2^{j} \int\left|x-k 2^{-j}\right|^{s+s^{\prime}} \frac{\left(\left|x-k 2^{-j}\right|+\left|k 2^{-j}\right|\right)^{-s^{\prime}}}{\left(1+\left|2^{j} x-k\right|\right)^{q}} d x .
\end{aligned}
$$

Using the coarse majoration

$$
\left(\left|x-k 2^{-j}\right|+\left|k 2^{-j}\right|\right)^{-s^{\prime}} \leq C\left(2^{-j}\left(1+\left|2^{j} x-k\right|\right)(1+|k|)\right)^{-s^{\prime}}
$$

INRIA 
one writes

$$
\begin{aligned}
\left|d_{j, k}\right| & \leq C 2^{j} \int\left|x-k 2^{-j}\right|^{s+s^{\prime}} \frac{\left(1+\left|2^{j} x-k\right|\right)^{-s^{\prime}}(1+|k|)^{-s^{\prime}}}{\left(1+\left|2^{j} x-k\right|\right)^{q}} d x \\
& \leq C 2^{j} \int \frac{\left|x-k 2^{-j}\right|^{s+s^{\prime}}}{\left(1+\left|2^{j} x-k\right|\right)^{s+s^{\prime}}} \frac{\left(1+\left|2^{j} x-k\right|\right)^{-s^{\prime}}}{\left(1+\left|2^{j} x-k\right|\right)^{-s^{\prime}}} \frac{(1+|k|)^{-s^{\prime}} 2^{-j s^{\prime}} d x}{\left(1+\left|2^{j} x-k\right|\right)^{q-\left(s+s^{\prime}\right)+s^{\prime}}}
\end{aligned}
$$

Remarking that $\frac{\left|x-k 2^{-j}\right|}{\left(1+\left|2^{j} x-k\right|\right)} \leq 2^{-j}$, this implies

$$
\begin{aligned}
\left|d_{j, k}\right| & \leq C 2^{-j\left(s+s^{\prime}\right)} 2^{-j s^{\prime}} \int \frac{(1+|k|)^{-s^{\prime}}}{\left(1+\left|2^{j} x-k\right|\right)^{q-\left(s+s^{\prime}\right)+s^{\prime}}} 2^{j} d x \\
& \leq C 2^{-j s}(1+|k|)^{-s^{\prime}} \int_{-\infty}^{+\infty} \frac{1}{(1+|u-k|)^{q-\left(s+s^{\prime}\right)+s^{\prime}}} d u .
\end{aligned}
$$

Let us choose $q$ such that $q-\left(s+s^{\prime}\right)+s^{\prime}>2$. The above integral is now well-defined and independent of $k$. Thus

$$
\left|d_{j, k}\right| \leq C 2^{-j s}(1+|k|)^{-s^{\prime}}
$$

which gives $f \in C_{0}^{s, s^{\prime}}$.

2. $C_{0}^{s, s^{\prime}} \subset K_{0}^{s, s^{\prime}}$

Let us move to the converse implication, which is more intricate, and for that, take a function $f$ belonging to the space $C_{0}^{s, s^{\prime}}$.

If $m<s+s^{\prime}<m+1$, then we know that $f$ admits a derivative of order $m$ around $x_{0}=0$. Then we change the notations and denote by $f$ its derivative of order $m$, and by $s$ the real number $s-m$. The problem is reduced to prove that, if $f \in C_{0}^{s, s^{\prime}}$ with $0<s+s^{\prime}<1$, there exists a polynomial $P$ of order less than $[s]$ such that

$$
\left|\frac{f(x)-P(x)}{|x|^{[s]}}-\frac{f(y)-P(y)}{|y|^{[s]}}\right| \leq C|x-y|^{s+s^{\prime}}(|x-y|+|x|)^{-s^{\prime}-[s]} .
$$

For any continuous function $g \in C_{0}^{s}$, let us denote by $T(g)$ the polynomial of order $[s]$ such that (1) holds at $x_{0}=0 . T$ is obviously a linear operator. We know that $C_{0}^{s, s^{\prime}} \subset C_{0}^{s}$, thus $f \in C_{x_{0}}^{s}$ admits such a polynomial, and $T(f)$ is well-defined.

Let $x, y$ be two real numbers. One can obviously assume that $|y| \leq|x|$, without loss of generality. Moreover, by replacing $x \rightarrow f(x)$ by $x \rightarrow f(-x)$, one can assume $x>0$. One can also make the assumption $y>0$ to simplify the proof. Indeed, if $y=0,(36)$ reduces to $|f(x)-P(x)| \leq C|x|^{s}$, which is obvious since $f \in C_{0}^{s, s^{\prime}} \subset C_{0}^{s}$. If $y<0$, one has

$$
\left|\frac{f(x)-P(x)}{|x|^{[s]}}-\frac{f(y)-P(y)}{|y|^{[s]}}\right| \leq\left|\frac{f(x)-P(x)}{|x|^{[s]}}-\frac{f(-y)-P(-y)}{|-y|^{[s]}}\right|
$$




$$
+\left|\frac{f(-y)-P(-y)}{|-y|^{[s]}}-\frac{f(y)-P(y)}{|y|^{[s]}}\right| .
$$

The second term, using $C_{0}^{s, s^{\prime}} \subset C_{0}^{s}$, is obviously smaller than $|-y|^{s-[s]}$, and

$$
\begin{aligned}
|-y|^{s-[s]} & \leq|x|^{s-[s]} \\
& \leq|x|^{s+s^{\prime}}|x|^{-s^{\prime}-[s]} \\
& \leq|x-y|^{s+s^{\prime}}(|x-y|+|x|)^{-s^{\prime}-[s]}
\end{aligned}
$$

which is the required bound for $\left|\frac{f(x)-P(x)}{|x|^{[s]}}-\frac{f(y)-P(y)}{|y|^{[s]}}\right|$. Now, to achieve the proof of Theorem 3.1, it is sufficient to prove that, for a polynomial $P$,

$$
\left|\frac{f(x)-P(x)}{|x|^{[s]}}-\frac{f(-y)-P(-y)}{|-y|^{[s]}}\right| \leq C|x-(-y)|^{s+s^{\prime}}(|x-(-y)|+|x|)^{-s^{\prime}-[s]}
$$

where $0<-y<x$.

Hence $0<|x-y|<x$, and $|x| \leq|x-y|+|x| \leq|x|$. We will also use the integer $j_{1}$ defined by

$$
2^{-j_{1}-1} \leq|x|<2^{-j_{1}}
$$

We will show that the polynomial $T(f)$ satisfies the good conditions to be the polynomial $P$ we are looking for to obtain (9). First one decomposes the term to study into two simpler terms

$$
\left|\frac{f(x)-P(x)}{|x|^{[s]}}-\frac{f(y)-P(y)}{|y|^{[s]}}\right| \leq(I)+(I I)
$$

with

$$
\begin{aligned}
(I) & =|f(y)-P(y)|\left|\frac{1}{|x|^{[s]}}-\frac{1}{|y|^{[s]}}\right|, \\
(I I) & =\left|\frac{f(x)-P(x)}{|x|^{[s]}}-\frac{f(y)-P(y)}{|x|^{[s]}}\right| \\
& =|x|^{-[s]}|(f(x)-P(x))-(f(y)-P(y))| .
\end{aligned}
$$

Let us study $(I)$. If $[s]=0,(I)=0$, hence we focus on the case $[s] \geq 1 . f \in C_{0}^{s}$ gives $|f(y)-P(y)| \leq C|y|^{s}$ (remember that $P$ has been chosen for that), thus

$$
\begin{aligned}
(I) & \leq C|y|^{s} \frac{|x|^{[s]}-|y|^{[s]}}{|x y|^{[s]}} \\
& \leq C|y|^{s} \frac{|x-y||x|^{[s]-1}}{|x y|^{[s]}} \\
& \leq C|y|^{s-[s]}|x|^{-1}|x-y| \\
& \leq C|x-y||x|^{s-[s]-1}
\end{aligned}
$$

INRIA 
A crucial point here, and in the following, is that

$$
|x-y||x|^{s-[s]-1} \leq|x-y|^{s+s^{\prime}}(|x-y|+|x|)^{-s^{\prime}-[s]} .
$$

Indeed,

$$
\begin{aligned}
|x-y||x|^{s-[s]-1} & \leq|x-y|^{s+s^{\prime}}|x|^{-s^{\prime}-[s]}\left(\frac{|x-y|}{|x|}\right)^{1-\left(s+s^{\prime}\right)} \\
& \leq C|x-y|^{s+s^{\prime}}|x|^{-s^{\prime}-[s]} \\
& \leq C|x-y|^{s+s^{\prime}}(|x-y|+|x|)^{-s^{\prime}-[s]}
\end{aligned}
$$

since $1 / 2(|x-y|+|x|) \leq|x| \leq(|x-y|+|x|)$. This is the required bound for $(I)$.

Let us now move to the last term (II).

Consider the development of $f$ on the wavelet basis

$$
f(x)=\sum_{j} \sum_{k} d_{j, k} \psi\left(2^{j} x-k\right)=\sum_{j} f_{j}(x),
$$

where $f_{j}(x)=\sum_{k} d_{j, k} \psi\left(2^{j} x-k\right)$ can be compared to the $j$-th term of a Littlewood-Paley expansion of $f$. It is easily shown that each $f_{j}$ is infinitely differentiable, and one can work with the polynomials $T\left(f_{j}\right)$.

Moreover, using the localization of the wavelet and its derivatives, one has

$$
\forall j, \quad \forall n, \quad\left|f_{j}^{(n)}(x)\right| \leq C 2^{j(n-s)}\left(1+2^{j}|x|\right)^{-s^{\prime}} .
$$

One decomposes $(I I)$ into

$$
(I I)=(I I I)+(I V)+(V)
$$

where

$$
\begin{aligned}
(I I I) & =|x|^{-[s]} \sum_{j \leq j_{1}}\left|\left(f_{j}(x)-T\left(f_{j}\right)(x)\right)-\left(T\left(f_{j}\right)(y)-T\left(f_{j}\right)(y)\right)\right|, \\
(I V) & =|x|^{-[s]} \sum_{j \geq j_{1}+1}\left|f_{j}(x)-f_{j}(y)\right|, \\
(V) & =|x|^{-[s]} \sum_{j \geq j_{1}+1}\left|T\left(f_{j}\right)(x)-T\left(f_{j}\right)(y)\right|
\end{aligned}
$$

One first studies the terms $(V)$, that contains only the polynomials $T\left(f_{j}\right)$. Let us remark that if $[s]=0,(V)=0$. We thus focus on the case $[s] \geq 1$. By definition, each $T\left(f_{j}\right)$ is the Taylor expansion of $f_{j}$ around 0 , thus

$$
T\left(f_{j}\right)(x)=\sum_{n=0}^{[s]} \frac{f_{j}^{(n)}(0)}{n !} x^{n} .
$$


Applying (41) and (40) for $x=0$,

$$
\begin{aligned}
(V) & \leq C|x|^{-[s]} \sum_{j \geq j_{1}+1} \sum_{n=1}^{[s]} 2^{j(n-s)}\left|x^{n}-y^{n}\right| \\
& \leq C|x|^{-[s]} \sum_{n=1}^{[s]}\left|x^{n}-y^{n}\right| \sum_{j \geq j_{1}+1} 2^{j(n-s)} \\
& \leq C|x|^{-[s]} \sum_{n=1}^{[s]}|x-y||x|^{n-1} \sum_{j \geq j_{1}+1} 2^{j(n-s)}
\end{aligned}
$$

Remarking that $n-s<0$, one has

$$
\begin{aligned}
(V) & \leq C|x-y||x|^{-[s]-1} \sum_{n=1}^{[s]} 2^{j_{1}(n-s)}|x|^{n} \\
& \leq C|x-y \| x|^{-[s]-1} 2^{-j_{1} s}\left(\sum_{n=1}^{[s]}\left(2^{j_{1}}|x|\right)^{n}\right)
\end{aligned}
$$

But $2^{j_{1}}|x|<1$. Hence

$$
\begin{aligned}
(V) & \leq C|x-y||x|^{-[s]-1} 2^{-j_{1} s}\left([s] 2^{j_{1}}|x|\right) \\
& \leq C|x-y||x|^{-[s]} 2^{j_{1}(1-s)} \\
& \leq C|x-y||x|^{s-[s]-1}
\end{aligned}
$$

which gives the required bound using (38).

Moving to $(I V)$, one sees that

$$
\begin{aligned}
(I V) & \leq C|x|^{-[s]} \sum_{j \geq j_{1}+1}|x-y| \sup _{z \in[y, x]}\left|f_{j}^{\prime}(z)\right| \\
& \leq C|x|^{-[s]}|x-y| \sum_{j \geq j_{1}+1} 2^{j(1-s)}\left(1+2^{j}|x|\right)^{-s^{\prime}}
\end{aligned}
$$

$s^{\prime}<0$ and $2^{j}|x| \geq 1$, thus $\left(1+2^{j}|x|\right)^{-s^{\prime}} \leq C\left(2^{j}|x|\right)^{-s^{\prime}}$. Then,

$$
\begin{aligned}
(I V) & \leq C|x-y||x|^{-s^{\prime}-[s]} \sum_{j \geq j_{1}+1} 2^{j\left(1-\left(s+s^{\prime}\right)\right)} \\
& \leq C|x-y||x|^{-s^{\prime}-[s]} 2^{j_{1}\left(1-\left(s+s^{\prime}\right)\right)} \\
& \leq C|x-y||x|^{s-[s]-1} \\
& \leq C|x-y|^{s+s^{\prime}}(|x-y|+|x|)^{-s^{\prime}-[s]}
\end{aligned}
$$


where we have used one more time (38).

Eventually we treat the term (III) by applying the Taylor formula

$$
\begin{aligned}
f_{j}(x) & =T\left(f_{j}\right)(x)+\frac{x^{[s]+1}}{[s] !} \int_{0}^{1} f_{j}^{([s]+1)}(u x)(1-u)^{[s]} d u \\
f_{j}(y) & =T\left(f_{j}\right)(y)+\frac{y^{[s]+1}}{[s] !} \int_{0}^{1} f_{j}^{([s]+1)}(u y)(1-u)^{[s]} d u,
\end{aligned}
$$

For sake of simplicity, we introduce the notation $g_{j}=f_{j}-T\left(f_{j}\right)$. Thus, for each $j$, one has

$$
\begin{aligned}
\left|g_{j}(x)-g_{j}(y)\right| \leq & C \mid x^{[s]+1} \int_{0}^{1} f_{j}^{([s]+1)}(u x)(1-u)^{[s]} d u \\
& -y^{[s]+1} \int_{0}^{1} f_{j}^{([s]+1)}(u y)(1-u)^{[s]} d u \mid \\
\leq & C\left|x^{[s]+1}-y^{[s]+1}\right|\left|\int_{0}^{1} f_{j}^{([s]+1)}(u x)(1-u)^{[s]} d u\right| \\
& +C|y|^{[s]+1} \int_{0}^{1}\left|f_{j}^{([s]+1)}(u x)-f_{j}^{([s]+1)}(u y)\right|(1-u)^{[s]} d u \\
\leq & C|x-y||x|^{[s]} \int_{0}^{1} \sup _{z \in[0, x]}\left|f_{j}^{([s]+1)}(z)\right|(1-u)^{[s]} d u \\
& +C|y|^{[s]+1} \int_{0}^{1}|u x-u y| \sup _{z \in[0, x]}\left|f_{j}^{([s]+2)}(z)\right|(1-u)^{[s]} d u \\
\leq & C|x-y \| x|^{[s]} 2^{j([s]+1-s)}\left(1+2^{j}|x|\right)^{-s^{\prime}} \\
& +C|y|^{[s]+1}|x-y| 2^{j([s]+2-s)}\left(1+2^{j}|x|\right)^{-s^{\prime}}
\end{aligned}
$$

Now, using that $\left(1+2^{j}|x|\right)^{-s^{\prime}} \leq\left(1+2^{j_{1}}|x|\right)^{-s^{\prime}} \leq C$ for $j \leq j_{1}$, one writes

$$
\begin{aligned}
\sum_{j \leq j_{1}} 2^{j([s]+1-s)}\left(1+2^{j}|x|\right)^{-s^{\prime}} & \leq C \sum_{j \leq j_{1}} 2^{j([s]+1-s)} \\
& \leq C 2^{j_{1}([s]+1-s)} \\
& \leq C|x|^{s-[s]-1} .
\end{aligned}
$$

Using the same method,

$$
\sum_{j \leq j_{1}} 2^{j([s]+2-s)}\left(1+2^{j}|x|\right)^{-s^{\prime}} \leq C|x|^{s-[s]-2} .
$$

One can now bound $(I I I)$

$$
(I I I)=|x|^{-[s]} \sum_{j \leq j_{1}}\left|g_{j}(x)-g_{j}(y)\right|
$$

$\mathrm{RR} \mathrm{n}^{\circ} 4545$ 


$$
\begin{aligned}
\leq & C|x|^{-[s]}|x-y||x|^{[s]}|x|^{s-[s]-1} \\
& +C|x|^{-[s]}|y|^{[s]+1}|x-y||x|^{s-[s]-2} \\
\leq & C|x-y||x|^{s-[s]-1} .
\end{aligned}
$$

One ends up the proof using (38).

\section{Acknowledgments}

We are grateful to J.M. Bony for his help and his comments on this paper, and to the referee for several remarks that improved our text.

The algorithm has been implemented in Fraclab, a software toolbox avalaible at http://www-rocq.inria.fr/fractales/

\section{References}

[1] J. Beran. Statistics for long-memory processes. Chapman and Hall, 1994.

[2] J.M. Bony. Second microlocalization and propagation of singularities for semilinear hyperbolic equations. In Hyperbolic equations and related topics (Kata/Kyoto, 1984), pages 11-49, Boston, 1986. MA: Academic Press.

[3] K. Daoudi, J. Lévy Véhel, and Y. Meyer. Construction of continuous functions with prescribed local regularity. Constructive Approximation, 14(3):349-385, 1998.

[4] I. Daubechies. Orthonormal bases of compactly supported wavelets. Comm. Pure Appl. Math, 41, 1988.

[5] B. Guiheneuf and J. Lévy Véhel. 2-microlocal analysis and applications in signal processing. In International Wavelets Conference, Tangier, April 1998.

[6] G.H. Hardy. On Weierstrass's non-differentiable function. Trans. Am. Math. Soc., 17:301-325, 1916.

[7] S. Jaffard. Pointwise smoothness, two-microlocalization and wavelet coefficients. In Publ. Mat., volume 35(1), pages 155-168. Conference on Mathematical Analysis (El Escorial, 1989), 1991.

[8] S. Jaffard and Y. Meyer. Wavelet methods for pointwise regularity and local oscillations of functions. In Mem. Amer. Math. Soc., 123(587). 1996.

[9] K. Kolwankar and J. Lévy Véhel. A time domain characterization of the fine local regularity of functions. J. Fourier Analysis App., 8(4):319-334, 2002.

[10] Y. Meyer. Ondelettes et Opérateurs. Hermann, 1990.

[11] Y. Meyer. Wavelets, Vibrations and Scalings. CRM Monograph Series. American Mathematical Society, 1997.

[12] S. Seuret and J. Lévy Véhel. The local Hölder function of a continuous function. ACHA, to appear.

[13] K. Weierstrass. On continuous functions of a real argument that do not have a well-defined differential quotient. Mathematische Werke, pages 71-74, 1895. 


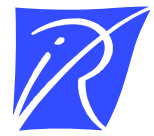

Unité de recherche INRIA Rocquencourt Domaine de Voluceau - Rocquencourt - BP 105 - 78153 Le Chesnay Cedex (France)

Unité de recherche INRIA Lorraine : LORIA, Technopôle de Nancy-Brabois - Campus scientifique 615, rue du Jardin Botanique - BP 101 - 54602 Villers-lès-Nancy Cedex (France)

Unité de recherche INRIA Rennes : IRISA, Campus universitaire de Beaulieu - 35042 Rennes Cedex (France)

Unité de recherche INRIA Rhône-Alpes : 655, avenue de l'Europe - 38330 Montbonnot-St-Martin (France)

Unité de recherche INRIA Sophia Antipolis : 2004, route des Lucioles - BP 93 - 06902 Sophia Antipolis Cedex (France)

INRIA - Domaine de Voluceau - Rocquencourt, BP 105 - 78153 Le Chesnay Cedex (France)

http://www.inria.fr

ISSN 0249-6399 